\title{
An Integral Representation and Bounds on the Effective Diffusivity in Passive Advection by Laminar and Turbulent Flows
}

\author{
Marco Avellaneda ${ }^{1 \star}$ and Andrew J. Majda ${ }^{2 \star \star}$ \\ ${ }^{1}$ Courant Institute, New York University, 251 Mercer Street, New York, NY 10012, USA \\ ${ }^{2}$ Department of Mathematics, and Program in Applied and Computational Mathematics, \\ Princeton University, Princeton, NJ 08544, USA
}

Received September 17, 1990

\begin{abstract}
Precise necessary and sufficient conditions on the velocity statistics for mean field behavior in advection-diffusion by a steady incompressible velocity field are developed here. Under these conditions, a rigorous Stieltjes integral representation for effective diffusivity in turbulent transport is derived. This representation is valid for all Péclet numbers and provides a rigorous resummation of the divergent perturbation expansion in powers of the Péclet number. One consequence of this representation is that convergent upper and lower bounds on effective diffusivity for all Peclet numbers can be obtained utilizing a prescribed finite number of terms in the perturbation series. Explicit rigorous examples of steady incompressible velocity fields are constructed which have effective diffusivities realizing the simplest upper or lower bounds for all Péclet numbers. A nonlocal variational principle for effective diffusivity is developed along with applications to advection-diffusion by random arrays of vortices. A new class of rigorous examples is introduced. These examples have an explicit Stieltjes measure for the effective diffusivity; furthermore, the effective diffusivity behaves like $\kappa_{0}(P e)^{1 / 2}$ in the limit of large Péclet numbers where $\kappa_{0}$ is the molecular diffusivity. Formal analogies with the theory of composite materials are exploited systematically.
\end{abstract}

\section{Introduction}

We are interested in the passive advection of a scalar quantity by a random, statistically homogeneous, incompressible, velocity field. This important problem arises commonly in the study of turbulent diffusion in the atmosphere [1], in dispersion of tracers in Bénard convection rolls [2] and porous materials [3], as

\footnotetext{
* Reseàrch partially supported by NSF DMS 90-05799 and ARO DAAL 03-89-K-0039 and AFOSR-90-0090

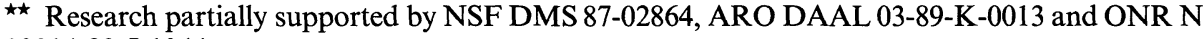
00014-89-J-1044
} 
well as in elementary models in plasma physics [4]. The equation for the advection-diffusion of a passive scalar is

$$
\frac{\partial T(x, t)}{\partial t}+\mathbf{u}(x, t) \cdot \nabla T(x, t)=\kappa_{0} \Delta T(x, t),
$$

where $\mathbf{u}(x, t)$ is a statistically homogeneous vector field satisfying

and

$$
\nabla \cdot \mathbf{u}(x, t)=0
$$

$$
\langle\mathbf{u}(x, t)\rangle=0,
$$

with brackets denoting statistical averaging. The constant $\kappa_{0}$ represents molecular diffusion. Despite the simplicity of (1), formal averaging of this equation with respect to the velocity statistics leads to

$$
\frac{\partial\langle T\rangle}{d t}+\langle\mathbf{u} \cdot \nabla T\rangle=\kappa_{0} \Delta\langle T\rangle,
$$

and hence to the nontrivial problem of determining the asymptotic behavior at large times of the statistically nonlinear term $\langle\mathbf{u} \cdot \nabla T\rangle$.

The question of long-time, large-scale asymptotics of the passive advection equation is particularly interesting due to the fact that it exhibits a crossover phenomenon: the statistical properties of $\mathbf{u}(x, t)$ and, in particular, the nature of the power-energy spectrum $\left\langle|\hat{\mathbf{u}}(k, \omega)|^{2}\right\rangle$ at low wave numbers $|k|$ can lead to various completely different space-time scaling laws and effective equations for (1) [5-7]. We shall say that the advection-diffusion Eq. (1) has mean-field behavior if the following property holds: given any smooth, compactly supported initial profile $T_{0}(x)$, the solution $T_{\delta}(x, t)$ of the initial-value problem

$$
\left\{\begin{aligned}
\frac{\partial T_{\delta}(x, t)}{\partial t}+\mathbf{u}(x, t) \cdot \nabla & T_{\delta}(x, t)=\kappa_{0} \Delta T_{\delta}(x, t) \\
T_{\delta}(x, 0) & =T_{0}(\delta x)
\end{aligned}\right.
$$

satisfies

$$
\lim _{\delta \downarrow 0} T_{\delta}\left(\frac{x}{\delta}, \frac{t}{\delta^{2}}\right)=\bar{T}(x, t)
$$

for almost all realizations of $\mathbf{u}(x)$, where $\bar{T}(x, t)$ is the solution of an appropriate diffusion equation

$$
\frac{\partial \bar{T}}{\partial t}(x, t)=\sum_{i j=1}^{d} \kappa_{i j}^{*} \frac{\partial^{2} \bar{T}(x, t)}{\partial x^{i} \partial x^{j}} .
$$

The corresponding tensor $\underset{i j}{*}$ is the effective diffusivity tensor, describing the macroscopic dispersion of the scalar $T_{\delta}$.

For example, in the simple limiting case of a statistically homogeneous, purely time-dependent velocity field $\mathbf{u}=\mathbf{u}(t)$, it is well known [8,9] that the crossover between Fickian and anomalous regimes depends on whether the Kubo diffusivity

$$
\delta \underset{\sim}{\kappa}=\int_{0}^{\infty}\left\langle\mathbf{u}(t) \mathbf{u}(0)^{T}\right\rangle d t,
$$


is finite or not. If $\delta \underset{\sim}{\kappa}$ is finite, the corresponding effective diffusivity tensor is given by

$$
\underset{\sim}{\kappa}=\kappa_{0} \mathbf{I}+\delta \underset{\sim}{\kappa}=\kappa_{0} \mathbf{I}+\int_{0}^{\infty}\left\langle\mathbf{u}(t) \mathbf{u}(0)^{T}\right\rangle d t
$$

Here, we present a mathematically rigorous study of the effective diffusivity for advection-diffusion with incompressible velocity fields that are time-independent, of the form $\mathbf{u}=\mathbf{u}(x)$. In contrast to the purely time-dependent case described in (9), with rapid decorrelation in time, for time-independent incompressible velocity fields, $\mathbf{u}(x)$, the correlation time is infinite. In this paper we discuss the mean field regime, so that the usual diffusive scaling (6) applies with an appropriate effective diffusion equation with the form in (7). A very natural non-dimensional characterization of the strength of the incompressible transport by $\mathbf{u}(x)$ compared with the molecular diffusivity, $\kappa_{0}$, is given by the Péclet number,

$$
P e=\frac{1}{\kappa_{0}}\left[\int \frac{\left\langle|d \hat{\mathbf{u}}(k)|^{2}\right\rangle}{|k|^{2}}\right]^{1 / 2},
$$

where $\left\langle|d \hat{\mathbf{u}}(k)|^{2}\right\rangle$ is the energy spectrum of the corresponding stationary incompressible velocity field $\mathbf{u}(x)$. One contribution in this paper is a proof that the finiteness of the Péclet number defined in (10) is an essentially necessary and sufficient condition to guarantee the mean-field behavior described in (7), (8) above. Explicit examples of the authors rigorously demonstrate that when the integral in (10) diverges, completely different anomalous scalings occur with nonlocal effective diffusion equations [7]. The proof of mean-field behavior when the Péclet number in (10) is finite is briefly discussed in Sect. 2 and carried out in the Appendix. Our proof uses the powerful homogenization methods developed by Papanicolaou and Varadhan [10], Tartar [11], and others [12, 13, 14] where the mean-field behavior is established under more stringent assumptions on $\mathbf{u}(x)$ than finiteness of the Péclet number. Furthermore, in Sect. 2, we prove that the homogenization formulas $[9,10]$ for the effective diffusivity tensor $\left(\kappa_{i j}^{*}\right)$ remain valid under the assumption that the integral in (10) is finite. For the remainder of the discussion here as well as for the rest of this paper, we always assume that the Péclet number defined in (10) is finite.

Following the discussion of mean field behavior in Sect. 2, the main new results are presented in Sects. 3-6. In Sect. 3, we derive a Stieltjes measure representation formula for the effective diffusivity. This tensor-valued measure is completely determined by the flow $\mathbf{u}(x)$. Furthermore, the Stieltjes measure remains invariant under the transformation $\mathbf{u}(x) \leftrightarrow \lambda \mathbf{u}(x)$ for any real $\lambda$ and contains the essential statistical/dynamical information about the flow that determines the effective diffusivity as a function of $\kappa_{0}$ and $P e$. In most applications, the Péclet number is very large and one advantage of the Stieltjes representation is that it remains valid at arbitrarily large Péclet numbers or arbitrarily small values of the molecular diffusivity, $\kappa_{0}$. In Sect. 3 , we develop some simple consequences of the representation formula including a precise characterization of the radius of convergence of the small Péclet number perturbation expansion as well as a direct link between properties of the effective diffusivity for $P e \uparrow \infty$ and/or $\kappa_{0} \downarrow 0$ and the behavior of the Stietljes measure in a neighborhood of zero. Besides many direct physical applications, the advection-diffusion by a steady incompressible velocity 


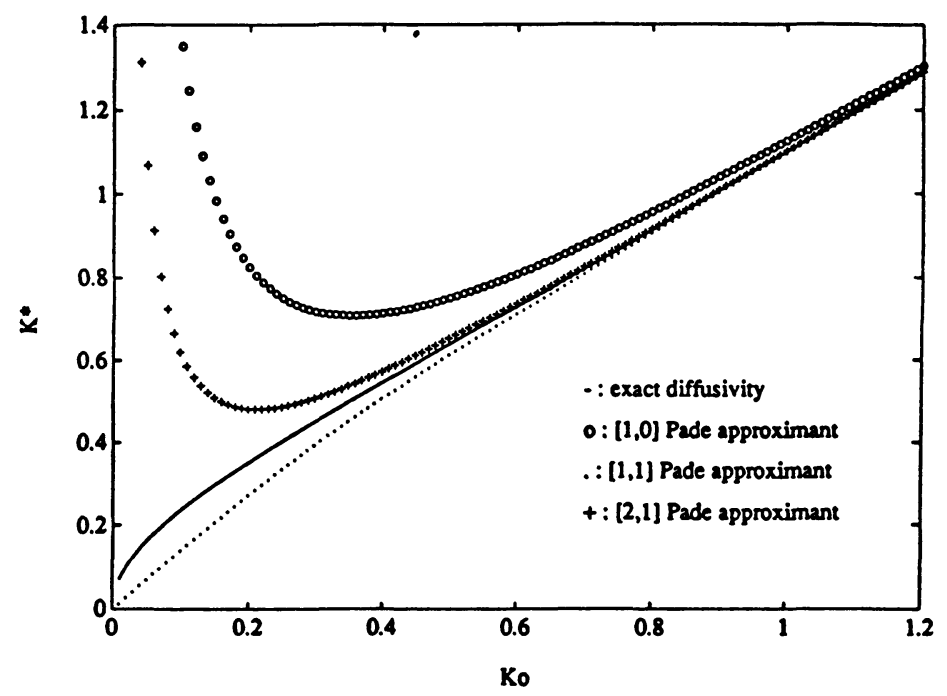

Fig. 1. The continuous curve represents the exact effective diffusivity $\kappa^{*}=$ $\sqrt{\frac{\kappa_{0}^{2}+\kappa_{0} \sqrt{1+\kappa_{0}^{2}}}{2}}$, as a function of the molecular diffusivity, $\kappa_{0}$, for the flow of Sect. VI with stream function $\psi_{0}$. The three discontinuous curves represent the corresponding first three Padé approximants. The Péclet number of this flow is $P e=1 / 2 \kappa_{0}$

field with mean-field behavior in the limit as $P e \uparrow \infty$ has an important role as a simple model problem for testing renormalized perturbation theories for turbulence $[15,16]$. The Stieltjes representation formula provides a rigorous resummation of the divergent perturbative series in $P e$. These applications are discussed at the end of Sect. 3.

In Sect. 4, we develop a rigorous theory of bounds for the effective diffusivity by utilizing the Stieltjes measure representation derived in Sect. 3. With this formula, we directly apply the theory of Pade approximants [17] to obtain rigorous upper and lower bounds which are valid for all Péclet numbers and depend on a finite number of moments in the perturbation series for small Péclet numbers. Furthermore, as the number of prescribed moment increases, the upper and lower bounds converge to the effective diffusivity for fixed, arbitrarily large Péclet number. The simplest special case of this theory yields the upper bound

$$
\frac{\kappa^{*}}{\kappa_{0}} \leqq 1+\frac{1}{d}(P e)^{2}
$$

for velocity fields $\mathbf{u}(x)$ with isotropic statistics with $d=$ the space dimension. The bound in (11) provides a rigorous proof that first order perturbation theory gives an upper bound for effective diffusivity valid for all Péclet numbers.

In Fig. 1 we graph the first upper and lower bounds as well as the actual effective diffusivity for a specific example (described in Sect. 6) to illustrate the potential usefulness of these bounds at moderate Péclet numbers. In Sect. 4, we 
also discuss the realizability of the bounds by suitable flows and present several concrete examples of incompressible steady velocity fields with the integral in (10) finite which have an effective diffusivity which achieves either the simplest upper or lower bounds for all Péclet numbers. We emphasize here that the concrete flows constructed in Sect. 4 are probably unphysical and have no relation to actual hydrodyamic flow fields. However, they serve the important purpose of showing that improvements in either upper or lower bounds must necessarily incorporate additional statistical information for actual fluid flows beyond the first few moments of the perturbation expansion.

In Sect. 5, we derive a nonlocal variational principle for the effective diffusivity. We also illustrate the potential utility of this variational method by applying it to obtain upper bounds on the diffusivity for incompressible velocity fields defined by suitable random arrays of vortices. In Sect. 6, we present a class of interesting nontrivial incompressible velocity fields where we can determine the Stieltjes measure explicitly. In one case, the effective diffusivity $\kappa^{*}$ is determined for all Péclet numbers by the formula,

$$
\kappa^{*}=\kappa_{0}\left(\frac{1+\left(1+4 P e^{2}\right)^{1 / 2}}{2}\right)^{1 / 2} .
$$

In particular, from (12) we have examples of flows where $\kappa^{*}$ behaves like $\kappa_{0}(P e)^{1 / 2}$ in the limit of large Péclet numbers. Such fractional power-law behavior at large Péclet numbers has been established through formal asymptotic or numerical calculations by several authors [2, 18, 43, 44] studying periodic arrays of convection rolls. In those works, it was shown that the boundary layers around separatrices between convection rolls give rise to the predicted enhanced diffusivity at large Péclet numbers. The examples in Sect. 6 involve singular velocities along a fractal separatrix, resulting in a similar enhancement mechanism. One of the authors will present elsewhere a substantial generalization of these examples, in which the effective diffusivity obeys power laws $\kappa^{*} \sim k_{0} P e^{\alpha}$ with exponents $\alpha \neq \frac{1}{2}$.

Throughout this paper, we have systematically exploited the formal analogy between properties of effective diffusivity for advective transport by a steadystate flow and the theory of composite materials.

Our study of diffusivity via Padé approximants for a Stieltjes integral in Sect. 3 and 4 is inspired by the work of Golden and Papanicolaou [19], Bergman [20], and Milton [21] for two-phase composite materials, and the work of Battacharya, Gupta and Walker [22] on periodic flows. Wolynes [23] derived a closely related formalism to study the renormalized transport coefficients of mode-coupling systems near equilibrium and Kraichnan [24] studied non-rigorously, with Padé approximants, the eddy diffusivity for an equation related to (1) with a fictitious decay of probability. Our examples in Sect. 6 are in some respects motivated by work of Hashin and Shtrikman [25] and Schulgasser [26] on composite materials. An announcement of some of the results presented here is contained in a recent letter of the authors [27]. For the reader's convenience, we conclude this introduction with a table of contents for the remainder of this paper. 


\section{Table of Contents}

I. Statistics for the Incompressible Velocity Field . . . . . . . . . . . . . . . . . . . . 344

II. Homogenization and the Basic Equation Defining the Effective Diffusivity . . . . 345

III. The Stieltjes Integral Representation Formula . . . . . . . . . . . . . . . . . . 349

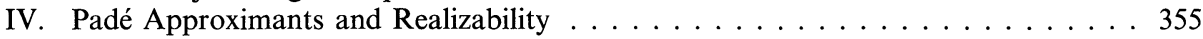

V. A Variational Principle for the Effective Diffusivity . . . . . . . . . . . . . 363

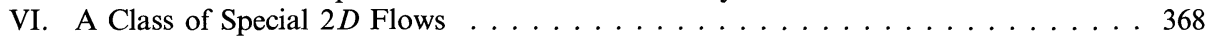

\section{Statistics for the Incompressible Velocity Field}

Here we briefly summarize and discuss the statistical assumptions which we make throughout this paper on the steady incompressible velocity field, $\mathbf{u}(x)$, in (1).

We represent formally such time-independent velocity fields as

$$
\mathbf{u}(x)=\int_{\mathbb{R}_{d}} e^{i k \cdot x} d \hat{\mathbf{u}}(k) ;
$$

where $d \hat{\mathbf{u}}(k)$ is the energy spectral measure, satisfying

$$
\begin{gathered}
\langle d \hat{\mathbf{u}}(k)\rangle=0, \quad k \in \mathbb{R}^{d}, \\
\left\langle d \hat{\mathbf{u}}(k) \otimes d \hat{\mathbf{u}}\left(k^{\prime}\right)\right\rangle=0, \quad \text { for } k \neq k^{\prime}, \\
k \cdot d \hat{\mathbf{u}}(k)=0 \quad \text { a.s. },
\end{gathered}
$$

reflecting the incompressibility condition (2), and

$$
\int_{\mathbb{R}_{d}}\left\langle|d \hat{\mathbf{u}}(k)|^{2}\right\rangle<\infty
$$

i.e., $\mathbf{u}(x)$ has finite kinetic energy. This formalism subsumes the cases of periodic or quasiperiodic flows, by suitably interpreting the averaging operation \langle\rangle as unit cell or Bohr averaging, respectively [10].

An essentially necessary and sufficient condition for (1) to have mean-field behavior, for time-independent fields $\mathbf{u}=\mathbf{u}(x)$, is [27] that

or, equivalently,

$$
\int_{\mathbb{R}_{d}} \frac{\left\langle|d \hat{\mathbf{u}}(k)|^{2}\right\rangle}{|k|^{2}}<\infty
$$

$$
\lim _{\delta \rightarrow 0} \int_{\mathbb{R}_{d}} \frac{\left\langle\mathbf{u}_{i}(x) \mathbf{u}_{i}(0)\right\rangle e^{-\delta|x|}}{|x|^{d-2}} d x<\infty .
$$

Conditions (1.6), (1.7) limit the strength of the velocity energy spectrum near $k=0$, or, equvalently, the long-range velocity correlation effects which favor large-scale advective motion. From (1.7) it follows, for instance, that power-law correlations $\left\langle\mathbf{u}(x) \mathbf{u}(0=\rangle \propto|x|^{-\gamma},|x| \gg 1\right.$, yield mean-field behavior for $\gamma>2$, and from (1.6) that periodic flows always satisfy mean-field behavior. A proof of the mean-field theorem under assumption (1.6) is given in the Appendix.

We will denote by $L^{p}(\langle\cdot\rangle)(1 \leqq p<\infty)$ the spaces of real-valued (or sometimes matrix-valued) functionals $\boldsymbol{\Phi}=\overline{\boldsymbol{\Phi}}[\mathbf{u}(\cdot)]$ of the random velocity field $\mathbf{u}$, which are measurable with respect to the $\sigma$-algebra generated by the sets $\{\mathbf{u}(x) \in A\}$, 
with $x \in \mathbb{R}^{d}$ and $A$ a Borel subset of $\mathbb{R}$, such that

$$
\|\boldsymbol{\Phi}\|_{L^{p}(\langle\cdot\rangle)} \equiv\left\langle|\boldsymbol{\Phi}|^{p}\right\rangle^{1 / p}<\infty .
$$

Another statistical assumption that we make, without loss of generality, is that $\mathbf{u}(x)$ is an ergodic random field, i.e. that the following property holds: for all measurable, real-valued functionals $\boldsymbol{\Phi}=\boldsymbol{\Phi}[\mathbf{u}(\cdot)]$ with the property that, for all $x \in \mathbb{R}^{d}$,

we have

$$
\boldsymbol{\Phi}[\mathbf{u}(x+\cdot)]=\boldsymbol{\Phi}[\mathbf{u}(\cdot)]
$$

$$
\Phi[\mathbf{u}(\cdot)]=\text { constant }=\langle\boldsymbol{\Phi}[\mathbf{u}(\cdot)]\rangle .
$$

This assumption is used in the proof of the mean-field theorem.

\section{Homogenization and the Basic Equation Defining the Effective Diffusivity}

The equation

$$
\frac{\partial T}{\partial t}+\mathbf{u}(x) \cdot \nabla T=\kappa_{0} \Delta T
$$

can be transformed into a divergence-form, parabolic, second-order equation, using the incompressibility of $\mathbf{u}(x)$ and the convergence of the integral $\frac{\left\langle|d \hat{\mathbf{u}}(k)|^{2}\right\rangle}{|k|^{2}}<\infty$, i.e. that the Péclet number is finite. This crucial condition will be always assumed hereafter. Such reduction is an important step in the proof of the mean-field behavior for (2.1). It is done by defining a stationary process $\mathbf{H}_{1}(x)$, taking values in the set of skew-symmetric matrices, given by

$$
\mathbf{H}_{1}(x)=\int e^{i k \cdot x} d \hat{\mathbf{H}}_{1}(k),
$$

where $d \hat{\mathbf{H}}_{1}(k)$ is a random measure such that

$$
\hat{\mathbf{H}}_{1}(\{0\})=0
$$

and, for $k \neq 0$,

$$
d \hat{\mathbf{H}}_{1}(k)=\frac{i}{|k|^{2}}[d \hat{\mathbf{u}}(k) \otimes \mathbf{k}-\mathbf{k} \otimes d \hat{\mathbf{u}}(k)] .
$$

From the incompressibility condition $(2), \mathbf{k} \cdot d \hat{\mathbf{u}}(k)=0$, we have

$$
i \mathbf{k} \cdot d \hat{\mathbf{H}}_{1}(k)=\frac{-1}{|k|^{2}}\left[\mathbf{k} \cdot d \hat{\mathbf{u}}(k) \mathbf{k}-|\mathbf{k}|^{2} d \hat{\mathbf{u}}(k)\right]=\hat{\mathbf{u}}(k),
$$

or, equivalently

Since

$$
\nabla \cdot \mathbf{H}_{1}(x)-\mathbf{u}(x) .
$$

$$
\operatorname{Trace}\left\langle\mathbf{H}_{1}^{T} \mathbf{H}_{1}\right\rangle=2 \int \frac{|d \hat{\mathbf{u}}(k)|^{2}}{|k|^{2}},
$$

$\mathbf{H}_{1}(x)$ is a well-defined, square-integable, stationary random process. From (2.3), (2.4), we have 


$$
\left\langle\mathbf{H}_{1}\right\rangle=0, \quad \mathbf{H}_{1}+\mathbf{H}_{1}^{T}=\mathbf{0} .
$$

Using the skew-symmetry of $\mathbf{H}_{1}$ we can rewrite (2.1) as

$$
\frac{\partial T(x, t)}{\partial t}=\nabla \cdot\left(\kappa_{0} \mathbf{I}-\mathbf{H}_{1}(x)\right) \cdot \nabla T(x, t) .
$$
The initial-value problem for the rescaled function $T_{\delta}(x, t)=T\left(\frac{x}{\delta}, \frac{t}{\delta^{2}}\right)$ is given
by

$$
\begin{cases}\frac{\partial T_{\delta}(x, t)}{\partial t}=\nabla \cdot\left(\kappa_{0} \mathbf{I}-\mathbf{H}_{1}\left(\frac{x}{\delta}\right)\right) \cdot \nabla T_{\delta}(x, t), & x \in D, t>0 \\ T_{\delta}(x, 0)=T_{0}(x), & x \in D, \\ T_{\delta}(x, 0)=0, & x \in \partial D, t>0,\end{cases}
$$

where $D$ is a bounded domain and $T_{0}$ is a smooth function supported in $D$. It is convenient to nondimensionalize the perturbation $\mathbf{H}_{1}(x)$ by setting

$$
\mathbf{H}(x) \equiv \frac{1}{\kappa_{0} P e} \mathbf{H}_{1}(x) .
$$

In particular, we have, from (2.7), $\operatorname{tr}\left\langle\mathbf{H}^{T} \mathbf{H}\right\rangle=2$. Equation (2.10) then becomes

$$
\left\{\begin{array}{lrl}
\frac{\partial T_{\delta}(x, t)}{\partial t}=\kappa_{0} \nabla \cdot\left(\mathbf{I}-P e \mathbf{H}\left(\frac{x}{\delta}\right)\right) \cdot \nabla T_{\delta}(x, t), & & x \in D, t>0 \\
T_{\delta}(x, 0)=T_{0}(x), & & x \in D, \\
T_{\delta}(x, t)=0, & \text { for } x \in \partial D,
\end{array}\right.
$$

and $P e$ can be viewed as a coupling parameter.

The homogenization theorem of Papanicolaou and Varadhan [10] applies to this initial value problem, assuming that the perturbation $\mathbf{H}(x)$ is uniformly bounded and stochastically continuous, so that mean-field behavior holds under these assumptions. These authors, following the general procedure of multiplescale asymtotics, postulated the ansatz

$$
T_{\delta}(x, t) \simeq \bar{T}(x, t)+\delta \underset{\sim}{\chi}\left(\frac{x}{\delta}\right) \nabla \bar{T}(x, t)+\cdots,
$$

where $\underset{\sim}{\chi}(x): \mathbb{R}^{d} \rightarrow \mathbb{R}^{d}$ is a random function such that $\mathbf{E}(x) \equiv \nabla \underset{\sim}{\underset{\sim}{\chi}}(x)+\mathbf{I}$ is a stationary random process satisfying the basic equations

$$
\nabla \times \mathbf{E}=0,
$$

and

$$
\left\langle|\mathbf{E}(x)|^{2}\right\rangle<\infty
$$


(We set, for $\eta \equiv\left(\eta_{i j}\right) ;|\eta|^{2} \equiv \sum_{i j} \eta_{i j} \eta_{j i}$.) It has been shown in [10] that problem

(i)-(iv) has a unique solution if $\mathbf{H}$ is essentially bounded; this is a consequence of the Lax-Milgram theorem. The function $\bar{T}(x, t)$ is chosen as the solution of the initial value problem for the operator

$$
\begin{aligned}
& \frac{\partial \bar{T}}{\partial t}(x, t)=\sum_{i j=1}^{d} \kappa_{i j}^{*} \frac{\partial^{2} \bar{T}(x, t)}{\partial x^{i} \partial x^{j}}, \quad x \in D, t>0, \\
& \bar{T}(x, 0)=T_{0}(x), \\
& \bar{T}(x, t)=0 \text { for } x \in \partial D, t>0,
\end{aligned}
$$

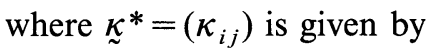

$$
\stackrel{\sim}{\kappa}^{*}=\kappa_{0}(\mathbf{I}+P e\langle\mathbf{H} \cdot \mathbf{E}\rangle) .
$$

This formula defines the effective diffusivity tensor ${\underset{\sim}{*}}^{*}$ corresponding to (2.1), so that one has

$$
\lim _{\delta \downarrow 0} T_{\delta}(x, t)=\bar{T}(x, t),
$$

in suitable sense. According to (2.19), and using the language of heat conduction, the vector $\stackrel{\sim}{*}^{*} \cdot \mathbf{v}$ represents, for any given $\mathbf{v} \in \mathbb{R}^{n}$, the average heat flux for unit time/unit volume corresponding to a steady state temperature gradient $\nabla T(x)=\mathbf{E}(\mathbf{x}) \cdot \mathbf{v}$, with average $\mathbf{v}$. A proof of the mean-field result for unbounded $\mathbf{H}(x)$ was given by $\mathbf{K}$. Oelschlager [13], under somewhat stringent assumptions on the density of the spectral measure $d \hat{\mathbf{u}}(k)$. Following references 10 and 13, we give in the Appendix a proof of the mean-field result (2.20) for fields $\mathbf{u}(x)$ and $\mathbf{H}(x)$ satisfying the higher integrability assumptions

and

$$
\left\langle|\mathbf{u}(x)|^{d / 2+\varepsilon}\right\rangle<+\infty
$$

$$
\left\langle|\mathbf{H}(x)|^{p}\right\rangle<+\infty
$$

for some $\varepsilon>0$, with $p=2+\varepsilon$ if $d=2$ and $p=d$ if $d \geqq 3$. In particular, the homogenization theorem is valid for arbitrary, incompressible, square-integrable Gaussian fields, satisfying the mean-field condition (1.6). For further details on the proof of (2.20) we refer the reader to the Appendix, and to [10-14], and concentrate hereafter on the properties of the effective diffusivity ${\underset{\sim}{*}}^{*}$.

The crucial ingredient for understanding the homogenization of (2.12) is to solve the basic Eqs. (2.14)-(2.17) yielding the field $\mathbf{E}(x)$ and hence the effective tensor $\stackrel{\sim}{*}^{*}$. For unbounded fields $\mathbf{H}(x)$, a solution to this problem can be constructed by approximation. More precisely, we set

$$
H_{i j}^{(n)}(x)=\left\{\begin{array}{lll}
H_{i j}(x) & \text { if } & \left|H_{i j}(x)\right| \leqq n \\
-n & \text { if } & H_{i j}(x)<-n \\
+n & \text { if } & H_{i j}(x)>n
\end{array}\right.
$$

We consider, for each $n \geqq 1$, the problems, 


$$
\begin{aligned}
& \nabla \cdot\left(\mathbf{I}+P e \mathbf{H}^{(n)}\right) \cdot \mathbf{E}^{(n)}=0, \\
& \nabla \times \mathbf{E}^{(n)}=0, \\
& \left\langle\mathbf{E}^{(n)}\right\rangle=\mathbf{I}, \\
& \left\langle\left|\mathbf{E}^{(n)}\right|^{2}\right\rangle<\infty .
\end{aligned}
$$

The corresponding solution $\mathbf{E}^{(n)}$ is unique, according to [10], and satisfies

$$
\left\langle\mathbf{F}^{T} \cdot\left(\mathbf{I}+P e \mathbf{H}^{(n)}\right) \cdot \mathbf{E}^{(n)}\right\rangle=0,
$$

for all square integrable fields $\mathbf{F}$ satisfying

$$
\langle\mathbf{F}\rangle=0, \quad \nabla \times \mathbf{F}=0 .
$$

Substituting $\mathbf{F}=\mathbf{E}^{(n)^{\prime}}=\mathbf{E}^{(n)}-\mathbf{I}$, we obtain

In particular,

$$
\begin{aligned}
0 & =\left\langle\mathbf{E}^{(n)^{\prime} T} \cdot(I+P e \mathbf{H}) \cdot \mathbf{E}^{(n)}\right\rangle \\
& \left.=\mathbf{E}^{(n)^{\prime} T} \cdot \mathbf{E}^{(n)^{\prime}}\right\rangle+P e\left\langle\mathbf{E}^{(n)^{\prime}} \cdot \mathbf{H}^{(n)}\right\rangle
\end{aligned}
$$

$$
\begin{aligned}
\operatorname{tr}\left\langle\mathbf{E}^{(n)^{\prime} T} \cdot \mathbf{E}^{(n)^{\prime}}\right\rangle & \leqq P e\left|\operatorname{tr}\left\langle\mathbf{E}^{(n)^{\prime}} \cdot \mathbf{H}^{(n)}\right\rangle\right| \\
& \leqq|P e|\left\langle\left|\mathbf{E}^{(n)^{\prime}}\right|^{2}\right\rangle^{1 / 2}\left\langle\left|\mathbf{H}^{(n)}\right|^{2}\right\rangle^{1 / 2}
\end{aligned}
$$

so that

and hence

$$
\left\langle\left|\mathbf{E}^{(n)^{\prime}}\right|^{2} \leqq P e^{2}\left\langle\left|\mathbf{H}^{(n)}\right|^{2}\right\rangle \leqq 2 P e^{2},\right.
$$

$$
\left\langle\left|\mathbf{E}^{(n)}\right|^{2}\right\rangle \leqq d+2 P e^{2} \text {. }
$$

By extracting if necessary a subsequence we conclude that

where E satisfies

$$
\mathbf{E}^{(n)} \rightarrow \mathbf{E} \quad \text { weakly in } L^{2}(\langle\cdot\rangle) \text {, }
$$

$$
\nabla \times \mathbf{E}=0, \quad\langle\mathbf{E}\rangle=I .
$$

Passing to the limit in (2.25), using the fact that, from the definition of $\mathbf{H}^{(n)}$, we have

we conclude that

$$
\lim _{n \rightarrow \infty}\left\langle\left|\mathbf{H}^{(n)}-\mathbf{H}\right|^{2}\right\rangle=0
$$

$$
\left\langle\mathbf{F}^{T} \cdot(\mathbf{I}+P e \mathbf{H}) \cdot \mathbf{E}\right\rangle=0,
$$

for all essentially bounded fields F satisfying (2.26). Hence

$$
\nabla \cdot(\mathbf{I}+P e \mathbf{H}) \cdot \mathbf{E}=0 .
$$

Moreover, from (2.30), we have

$$
\left\langle|\mathbf{E}|^{2} \leqq d+2 P e^{2} .\right.
$$

We have not been able to show that, if $\mathbf{H}$ is unbounded, the solution to Eq. (2.35) with the constraints $\nabla \times \mathbf{E}=0,\langle\mathbf{E}\rangle=\mathbf{I}$ is unique. Nevertheless, if we define $\kappa_{\tilde{\kappa}}^{*}$ by (2.19), then the mean-field result proved in the Appendix shows that the function $T_{\delta}$ solving (2.17) converges to the solution $\bar{T}$ of (2.18). This implies that the value of $\boldsymbol{\kappa}^{*}$ is independent of the particular subsequence of $\mathbf{E}^{(n)}$ used to define the field $\mathbf{E}$ and strongly suggests - but does not constitute a proof - that Eq. (2.35) with the constraints $\nabla \times \mathbf{E}=0,\langle\mathbf{E}\rangle=\mathbf{I}$ has a unique solution. 
Following Golden and Papanicolaou [19] we introduce a scattering-theory formalism and transform the basic problem (2.14)-(2.17) into an integral equation for the field $\mathbf{E}$. We consider the operator $\Gamma=\left(\Gamma_{i j}\right)$, given by

$$
\Gamma_{i j}=(-\Delta)^{-1} \frac{\partial^{2}}{\partial x^{i} \partial x^{j}}, \quad 1 \leqq i, j \leqq d,
$$

with Fourier symbols

$$
\begin{aligned}
\hat{\Gamma}_{i j}(k) & =-\frac{k_{i} k_{j}}{|k|^{2}}, & & \text { if } k \neq 0,1 \leqq i, j \leqq n, \\
& =0 & & \text { if } k=0 .
\end{aligned}
$$

The operator $-\boldsymbol{\Gamma}$ is equal to the projection onto curl-free, mean zero, square integrable fields. Equation (2.34) implies that $(I+P e \mathbf{H}) \cdot \mathbf{E}$ is orthogonal to all curl-free fields with zero mean. Hence,

$$
\boldsymbol{\Gamma}[(\mathbf{I}+P e \mathbf{H}) \mathbf{E}]=0,
$$

but, since $\boldsymbol{\Gamma} \mathbf{E}=\mathbf{I}-\mathbf{E}$, we have

$$
\mathbf{E}-P e \Gamma \mathbf{H E}=\mathbf{I} .
$$

This is the basic equation that we shall use in order to derive the Stieltjes integral representation formula. It is also useful to consider the equation satisfied by the fluctuating component of $\mathbf{E}, \mathbf{E}^{\prime}=\mathbf{E}-\mathbf{I}$, which is

$$
\mathbf{E}^{\prime}-P e \Gamma \mathbf{H E}^{\prime}=P e \Gamma \mathbf{H} .
$$

Such perturbation equations were considered by several authors, in the context of random media $[19,28]$.

\section{The Stieltjes Integral Representation Formula}

By analyzing the perturbation Eqs. (2.40) ands (2.41), we will show that to each incompressible, zero mean, time-independent velocity field $\mathbf{u}(x)$ with finite Péclet number, i.e., such that

$$
\int \frac{\left\langle|d \hat{\mathbf{u}}(k)|^{2}\right\rangle}{|k|^{2}}<\infty,
$$

there corresponds a unique positive definite, tensor-valued measure $d \mu(\tau)$ satisfying

$$
\mu([a, b])=\mu([-b,-a]), \quad \text { for } 0<a<b,
$$

such that the effective diffusivity $\stackrel{\sim}{*}^{*}=\kappa_{0}(\mathbf{I}+P e\langle\mathbf{H E}\rangle)$ is given by the representation formula

$$
\stackrel{\kappa}{\kappa}^{*}=\kappa_{0}\left(\mathbf{I}+\int_{-\infty}^{+\infty} \frac{P e^{2} d \mu(\tau)}{1+P e^{2} \tau^{2}}\right) .
$$

We also discuss several consequences of this result for moderate and large values of the Péclet number. 
III.1. The Derivation. Consider the sequence of linear operators $\mathbf{T}_{n}, n \in \mathbf{N}$ defined by

$$
\mathbf{T}_{n} \underset{\sim}{\phi}=\mathrm{i} \Gamma \mathbf{H}^{(n)} \phi \underset{\sim}{ }
$$

where $\boldsymbol{\Gamma}$ and $\mathbf{H}^{(n)}$ are defined in (2.37) and (2.23), respectively. For each $n$, the operator $\mathbf{T}_{n}$ is bounded and hermitian on the subspace of $L^{2}(\langle\cdot\rangle)$ defined by

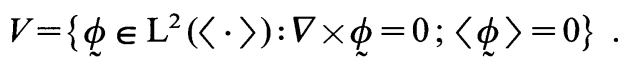

By the Spectral Theorem for bounded, hermitian operators in a Hilbert space [29], there exists an increasing family of projection operators $\left\{\mathbf{R}_{n}(\tau)\right\}$, $-\infty \leqq \tau \leqq \infty-$ the spectral resolution of $\mathbf{T}_{n}-$ such that

$$
\mathbf{R}_{n}(-\infty)=0, \quad \mathbf{R}_{n}(+\infty)=\mathbf{I}
$$

and such that, for all bounded, continuous functions $f(\tau): \mathbb{R} \rightarrow \mathbb{C}$, we have

$$
f\left(\mathbf{T}_{n}\right)=\int_{-\infty}^{+\infty} f(\tau) d \mathbf{R}_{n}(\tau)
$$

We apply this theorem to represent the solution of the equation

$$
\mathbf{E}^{(n)}-\operatorname{Pe} \boldsymbol{\Gamma} \mathbf{H}^{(n)} \mathbf{E}^{(n)}=\mathbf{I} .
$$

Noting that $\mathbf{E}^{(n)^{\prime}}=\mathbf{E}^{(n)}+\mathbf{I}$, with $\mathbf{E}^{(n)^{\prime}} \in V$, we write (3.8) as

$$
\mathbf{E}^{(n)^{\prime}}-P e \Gamma \mathbf{H}^{(n)} \mathbf{E}^{(n)^{\prime}}=P e \Gamma \mathbf{H}^{(n)} .
$$

The right-hand side of this equation can be regarded as an element of $V$.

Taking $f(\tau)=(1+i P e \tau)$, we obtain from (3.7)

$$
\mathbf{E}^{(n)^{\prime}}=\int_{-\infty}^{+\infty} \frac{\operatorname{Ped} \mathbf{R}_{n}(\tau)\left(\boldsymbol{\Gamma} \mathbf{H}^{(n)}\right)}{1+i \operatorname{Pe} \tau} .
$$

Consequently, we have

$$
\left\langle\mathbf{E}^{(n)^{\prime} T} \cdot \mathbf{E}^{(n)^{\prime}}\right\rangle=\int_{-\infty}^{+\infty} \frac{P e^{2} d \mu_{n}(\tau)}{1+P e^{2} \tau^{2}},
$$

where $\underset{\sim}{\mu_{n}}(\tau)$ is defined in terms of $\mathbf{H}^{(n)}$ and the spectral resolution $\mathbf{R}_{n}$, by the formula

$$
\mu_{n}(\tau)=\left\langle\mathbf{H}^{(n) \mathrm{T}} \cdot \boldsymbol{\Gamma} \cdot \mathbf{R}_{n}(\tau) \cdot \boldsymbol{\Gamma} \cdot \mathbf{H}^{(n)}\right\rangle
$$

The fact that $\mathbf{T}_{n}$ is hermitian implies that $\underset{\sim}{\underset{n}{\alpha}}$ satisfies (3.2). We thus conclude from (3.11) that

$$
\mathbf{I}+\left\langle\mathbf{E}^{(n)^{\prime} T} \cdot \mathbf{E}^{(n)}\right\rangle=\mathbf{I}+\int_{-\infty}^{+\infty} \frac{P e^{2} d \mu_{n}(\tau)}{1+P e^{2} \tau^{2}} .
$$

To obtain the representation for the effective diffusivity, we pass to the limit in this identity as $n \rightarrow \infty$. For this, notice that, from (2.24)-(2.27), we have

$$
\mathbf{I}+\left\langle\mathbf{E}^{(n)^{\prime} T} \cdot \mathbf{E}^{(n)^{\prime}}\right\rangle=\mathbf{I}+P e\left\langle\mathbf{H}^{(n)} \cdot \mathbf{E}^{(n)}\right\rangle
$$


and, since $\mathbf{E}^{(n)}$ converges along a subsequence to $\mathbf{E}$ and

$$
\lim _{n \rightarrow \infty}\left\langle\left|\mathbf{H}^{(n)}-\mathbf{H}\right|^{2}\right\rangle=0,
$$

the limit of the left-hand side (3.13) is given by

$$
\begin{aligned}
\lim _{n \rightarrow \infty}\left(I+P e\left\langle\mathbf{H}^{(n)} \cdot \mathbf{E}^{(n)^{\prime}}\right\rangle\right) & =I+P e\left\langle\mathbf{H} \cdot \mathbf{E}^{\prime}\right\rangle=I+\left\langle\mathbf{E}^{\prime T} \cdot \mathbf{E}^{\prime}\right\rangle \\
& =\mathbf{I}+P e\langle\mathbf{H} \cdot \mathbf{E}\rangle .
\end{aligned}
$$

On the other hand, from (3.6), (3.12), we have

$$
\begin{aligned}
\operatorname{Tr} \int_{-\infty}^{+\infty} d \mu_{n}(\tau) & =\operatorname{Tr}\left\langle\mathbf{H}^{(n)} \cdot \boldsymbol{\Gamma} \cdot \mathbf{H}^{(n)}\right\rangle \\
& \leqq\left\|\mathbf{H}^{(n)}\right\|_{L^{2}(\langle\cdot\rangle)}^{2} \\
& \leqq 2
\end{aligned}
$$

so, by Helly's lemma, $\left\{d \mu_{n}\right\}$ is a relatively compact set of measures. If $\mu$ is a limit point of $\left\{\underline{\mu}_{n}\right\}_{n \geq 1}$, we have, passing to the limit in the right-hand side of (3.13) and using (3.15),

$$
\mathbf{I}+P e\langle\mathbf{H E}\rangle=I+P e\left\langle\mathbf{H E}^{\prime}\right\rangle=\mathbf{I}+\int_{-\infty}^{+\infty} \frac{P e^{2} d \mu(\tau)}{1+P e^{2} \tau^{2}},
$$

which, after multiplication by $\kappa_{0}$, yields the desired representation formula (3.3) for the effective diffusivity $\kappa^{*}$. We show next that $d \mu(\tau)$ is determined completely from $\kappa^{*}$, i.e., that, given $\kappa_{0}$ and $\kappa^{*}$ as a function of the Péclet number $P e$, there is a unique measure $d \mu(\tau)$ satisfying (3.3). To see this, we consider the identity

$$
\frac{1}{\pi} \int_{-\infty}^{+\infty} \frac{e^{i s t} d s}{1+t^{2}}=e^{-|t|}
$$

Denoting by $\mathscr{F}$ and $\mathscr{L}$ the Fourier and Laplace transform operators,

$$
\mathscr{F}[\phi](t)=\int_{-\infty}^{+\infty} \phi(s) e^{i s t} d s ; \quad \mathscr{L}[\phi](t)=\int_{0}^{\infty} \phi(s) e^{-s t} d s,
$$

and using Plancherel's identity,

$$
\int_{-\infty}^{+\infty} \mathscr{F}[\phi](t) \overline{\mathscr{F}[\psi](t)} d t=2 \pi \int_{-\infty}^{+\infty} \phi(s) \overline{\psi(s)} d s,
$$

we have, for $\theta>0, P e>0$, 


$$
\begin{aligned}
\frac{1}{\kappa_{0} P e}\left(\kappa_{\sim}^{*}(P e)-\kappa_{0} \mathbf{I}\right) & =\int_{-\infty}^{+\infty} \frac{P e d \underset{\sim}{\mu}(\tau)}{1+P e^{2} \tau^{2}} \\
& =\frac{1}{2} \int_{-\infty}^{+\infty} e^{-|\theta| / P e} \mathscr{F}[\mu](\theta) d \theta \\
& =\int_{-\infty}^{\infty} e^{-|\theta| / P e} \mathscr{F}[\mu](\theta) d \theta \\
& =\mathscr{L} \circ \mathscr{F}[\mu]\left(\frac{1}{P e}\right) .
\end{aligned}
$$

Inversion of this identity (cf. Widder [30]) yields a formal relation determining $\underset{\sim}{\mu}$ in terms of the function $\underset{\sim}{*}(P e)$.

III.2. Expansion in Powers of $\mathrm{P}^{2}$ and Moments of the Representation Measure. A more useful relation between $\underset{\sim}{\mu}$ and $\mathbf{H}$ (and hence $\mathbf{u}$ ) can be obtained by equating the coefficients of the asymptotic expansion of the Stieltjes integral representation (3.3) in powers of $P e^{2}$ and those of the expansion of $\underset{\sim}{*}$ obtained by formally solving the equation (2.39), so that

$$
\mathbf{E}=(\mathbf{I}-\operatorname{Pe} \boldsymbol{\Gamma} \mathbf{H})^{-1} \mathbf{I},
$$

substituting this expression in (2.19), and expanding in Pe. Expanding (3.3) formally we obtain

$$
\frac{1}{\kappa_{0}}{\underset{\sim}{\kappa}}^{*}=\mathbf{I}+\sum_{n=1}^{\infty}(P e)^{2 n}(-1)^{n-1} \int_{-\infty}^{+\infty} \tau^{2(n-1)} d \underset{\sim}{\mu}(\tau)
$$

while the second procedure leads to

$$
\begin{aligned}
\frac{1}{\kappa_{0}}{\underset{\sim}{\kappa}}^{*} & =\left\langle\mathbf{I}+P e\left\langle\mathbf{H}(\mathbf{I}-P e \underset{\sim}{\Gamma} \mathbf{H})^{-1} \mathbf{I}\right\rangle\right\rangle \\
& =\mathbf{I}+\sum_{n=1}^{\infty}(P e)^{2 n}\left\langle\mathbf{H}(\boldsymbol{\Gamma} \mathbf{H})^{2 n-1}\right\rangle,
\end{aligned}
$$

where we used the fact that $\left\langle\mathbf{H}(\Gamma \mathbf{H})^{2 n}\right\rangle=0$, for $n \geqq 1$, since $\mathbf{H}$ is skew-symmetric. Equating coefficients, we obtain the formal relations between successive terms of both expansions:

$$
\int_{-\infty}^{+\infty} \tau^{2 N} d \underline{\sim}(\tau)=(-1)^{N}\left\langle\mathbf{H}(\boldsymbol{\Gamma} \mathbf{H})^{2 N+1}\right\rangle,
$$

for $N=0,1,2, \ldots$.

Such relations are immediately justified for bounded $\mathbf{H}$. In fact, the operator

$$
\mathbf{T}=\boldsymbol{\Gamma} \mathbf{H}
$$

is then bounded in $L^{2}(\langle\cdot\rangle)$ and thus the expansions (3.23), (3.24) converge for small enough $P e$. The justification of (3.25) for unbounded fields $\mathbf{H}(x)$ is more 
delicate since the operator $\boldsymbol{\Gamma} \mathbf{H}$ is unbounded in $L^{2}(\langle\cdot\rangle)$. For velocity fields with finite moments of all orders,

$$
\left\langle|\mathbf{H}(x)|^{N}\right\rangle<\infty, \quad N \geqq 1,
$$

the formulas (3.25) are nevertheless valid. This can be shown using the $L^{N}$ boundedness of the operator $\Gamma$ and the arguments developed by Oelschlager [13]. The reader is referred to that paper for details.

We will assume in the rest of the paper, for simplicity, that the identity (3.25) holds at least for $N=0$, i.e., that

$$
\int_{-\infty}^{+\infty} d \mu(\tau)=\langle\mathbf{H} \cdot \boldsymbol{\Gamma} \cdot \mathbf{H}\rangle
$$

Taking the trace of both sides of this equation we obtain

$$
\int_{-\infty}^{+\infty} \operatorname{tr} d \mu(\tau)=1
$$

using the fact that $\operatorname{tr}\langle\mathbf{H} \Gamma \mathbf{H}\rangle=\frac{1}{2} \operatorname{tr} \mathbf{H}^{T} \mathbf{H}=1$ (cf. Sect. 2). Thus, this assumption implies that the scalar measure $d v(\tau)=\operatorname{tr} d \mu(\tau)$ is a probability measure. Condition (3.27) holds for uniformly bounded $\mathbf{H}$. More generally, it can be shown, using the arguments of [13, Sect. 4], that (3.27) holds for $\mathbf{H} \in L^{4}(\langle\cdot\rangle)$.

In the following paragraphs we discuss some immediate consequences of the Stieltjes integral representation.

III.3. Radius of Convergence of the Expansion of $\kappa_{\sim}^{*}$ for Small Péclet Number. An important application of the Stieltjes integral representation is that it provides a formula for the effective diffusivity at large values of the Péclet number $P e$, determined by the coefficients of the perturbation expansion at small $P e$. This "analytic continuation" formula for $\kappa^{*}$ is particularly useful since the direct perturbation expansion (3.24) can have small, or even vanishing, radius of convergence. The radius of convergence, $\rho$, can be related to the representation measure $\underset{\sim}{\mu}$ in a simple way. In fact, we have

$$
\rho=\frac{1}{[1 . \text { u.b. supp } \underset{\sim}{\mu}]},
$$

where supp $\underset{\sim}{\mu}$ denotes the support of $\underset{\sim}{\mu}$, i.e. the smalles closed set such that $\underset{\sim}{\mu}\{(-\infty,+\infty) \backslash \operatorname{supp} \underset{\sim}{\mu}\}=0$. To see this, we notice that if $\frac{|P e|}{\rho}<1$, then

$$
\begin{aligned}
P e^{2(N+1)}\left|\int \tau^{2 N} d \mu(\tau)\right| & \leqq|P e|^{2} P e^{2 N}[1 \text {.u.b. supp } \mu]^{2 N} \\
& =|P e|^{2} \cdot\left|\frac{P e}{\rho}\right|^{2 N},
\end{aligned}
$$

for all $N$ so that the series (3.23) converges absolutely for $|P e|<\rho$. Also, for $|P e|>\rho$, we have that, for all $\varepsilon>0$, there exists a positive constant $C(\varepsilon)$ such that 


$$
\begin{aligned}
P e^{2(N+1)}\left|\int \tau^{2 N} d \mu \underline{\sim}(\tau)\right| & \geqq C(\varepsilon)|P e|^{2(N+1) \mid 1 . u . b . ~ s u p p} \underset{\sim}{\mu}-\left.\varepsilon\right|^{2 N} \\
& =C(\varepsilon)|P e|^{2}\left|\frac{P e}{\rho}(1-\varepsilon \rho)\right|^{2 N} .
\end{aligned}
$$

Choosing $\varepsilon$ sufficiently small, we see that the norms of the successive terms of (3.23) are bounded from below by $C(\varepsilon) \theta^{2 N}$, for some $\theta>1$, and hence that (3.23) is divergent. In particular, if $\operatorname{supp} \mu=(-\infty,+\infty)$, the radius of conver gence of (3.23) or (3.24) is equal to zero. Since the support of the measure $d \mu$ can be related to the spectral properties of $\boldsymbol{\Gamma} \mathbf{H}$ through (3.12), we can estimate the radius of convergence by

$$
\rho \leqq\|\boldsymbol{\Gamma} \mathbf{H}\|_{L^{2}(\langle\cdot\rangle), L^{2}(\langle\cdot\rangle)} .
$$

For uniformly bounded fields $\mathbf{H}$, we can further estimate the right-hand side of (3.32) using the fact that $\|\Gamma\|_{L^{2}(\langle\cdot\rangle), L^{2}(\langle\cdot\rangle)}=1$, to obtain finally

$$
\rho \leqq\|\mathbf{H}\|_{L^{\infty}(<\cdot>)}
$$

III.4. Behavior of $\kappa^{*}$ as $P e \rightarrow \infty$, or $\kappa_{0} \rightarrow 0$. In the remainder of this section we assume for simplicity that $\kappa_{\tilde{\alpha}}^{*}=\kappa^{*}$ I, i.e. that the flow is macroscopically isotropic. Fixing the value of $\kappa_{0}$ and letting $P e \rightarrow \infty$ in the representation formula

$$
\kappa^{*}=\kappa_{0}\left[1+\frac{1}{d} \int_{-\infty}^{+\infty} \frac{P e^{2} d v(\tau)}{1+P e^{2} \tau^{2}}\right],
$$

where $d \nu(\tau)=\operatorname{Tr} d \underset{\sim}{\mu}(\tau)$, we obtain

$$
\lim _{P e \rightarrow \infty} \frac{\kappa^{*}}{\kappa_{0}}=\frac{1}{d} \int_{-\infty}^{+\infty} \frac{d v(\tau)}{\tau^{2}}
$$

The right-hand side of this equation represents the maximum enhancement of the diffusivity that is possible by diffusion-advection by velocity fields $\lambda \mathbf{u}(x)$, as the (r.m.s.) velocity amplitude $\lambda$ increases, with a fixed value of the molecular diffusivity. Thus, the limit of $\kappa^{*}$ as $P e \rightarrow \infty$ is either infinite or equal to a nondimensional constant multiplied by the bare diffusivity $\kappa_{0}$. The constant, $\int_{-\infty}^{+\infty} \tau^{-2} d \nu(\tau)$, is determined explicity from the representation measure.

Another interesting limit arises when the amplitude of the flow field is held fixed while the diffusivity tends to zero. Recalling that

$$
P e^{2}=\frac{1}{\kappa_{0}^{2}} \int \frac{\left\langle|d \hat{\mathbf{u}}(k)|^{2}\right\rangle}{|k|^{2}},
$$

and substituting this expression in (3.34) we obtain

$$
\kappa^{*}=\kappa_{0}+\frac{1}{d} \int_{-\infty}^{+\infty} \frac{\kappa_{0} \alpha^{2} d v(\tau)}{\kappa_{0}^{2}+\alpha^{2} \tau^{2}}
$$

with $\alpha^{2}=\int \frac{\left\langle\left. d \hat{\mathbf{u}}(k)\right|^{2}\right\rangle}{|k|^{2}}$. Passing to the limit as $\kappa_{0}$ tends to zero in (3.36), we
obtain 


$$
\lim _{\kappa_{0} \rightarrow 0} \kappa^{*}=\frac{\pi}{d}\left(\int \frac{\left\langle|d \hat{\mathbf{u}}(k)|^{2}\right\rangle}{|k|^{2}}\right)^{1 / 2} \gamma
$$

with

$$
\gamma=\lim _{\tau \downarrow 0}\left[\frac{v(\tau)-v(-\tau)}{2 \tau}\right]
$$

For representation measures with continuous densities $d v / d \tau$ formula (3.37) becomes

$$
\lim _{\kappa_{0} \rightarrow 0} \kappa^{*}=\frac{d}{\pi}\left(\int \frac{\left\langle|d \hat{u}(k)|^{2}\right\rangle}{|k|^{2}}\right)^{1 / 2}\left(\frac{d v(0)}{d \tau}\right) .
$$

In particular, for $0<\frac{d v(0)}{d \tau}<\infty$, the limiting value is independent of $\kappa_{0}$. It seems reasonable to conjecture that $\lim _{\kappa_{0} \rightarrow 0} \kappa^{*}$ coincides with the eddy-diffusivity of inviscid transport computed by several authors for isotropic flows exhibiting highly ergodic or chaotic behavior [24,31]. In general, the numbers $\frac{d v(0)}{d \tau}$ and $\int_{-\infty}^{+\infty} \frac{d v(\tau)}{\tau^{2}}$, associated with the $\kappa_{0} \rightarrow 0$ and $P e \rightarrow \infty$ limits, respectively, are very difficult to compute. Unfortunately, they cannot be determined in any useful way by any finite set of moments of $d v(\tau)$.

In Sects. IV and VI we discuss some rigorous examples, corresponding to highly idealized flows, for which the representation measure can be computed explicitly.

\section{Padé Approximants and Realizability}

IV.1. General Principles. The Stieltjes representation formula (3.3) provides an expression for $\underset{\sim}{*}$ that remains valid for arbitrarily large values of the Péclet number. For simplicity, we will assume in the sequel that $\mathbf{u}(x)$ gives rise to an isotopic diffusivity, $\underset{\sim}{*}=\kappa^{*} \mathbf{I}$ which, by the arguments of the previous section, satisfies

$$
\kappa^{*}=\kappa_{0}\left(1+\frac{1}{d} \int_{-\infty}^{+\infty} \frac{P e^{2} d v(\tau)}{1+P e^{2} \tau^{2}}\right)
$$

with $v(\tau)=$ trace $\mu(\tau)$. In particular, from (3.27)-(3.28), $v(\tau)$ is the distribution function of a probability measure in $(-\infty,+\infty)$, i.e.

$$
\int_{-\infty}^{+\infty} d v(\tau)=1
$$

Integral expressions involving a positive measure, such as (4.1) appear in many branches of physics as a result of spectral perturbation calculations [17, 23]. Their approximations by rational functions, whenever feasible, have been extensively studied. For instance, the overall physical properties of a two-phase composite material, such as the dielectric constant or the electrical conductivity, can be studied using this approach. In that case the relevant perturbation parameter 
measures the contrast between the physical properties of the individual components. Several authors, including Bergman [20], Milton [21], and Golden and Papanicolaou [19], have successfully used integral representation formulas and rational function approximations to obtain information about the effective response of binary composites.

The idea behind the method of Padé approximants is to obtain upper and lower bounds on $\kappa^{*}$ by maximizing or minimizing the right-hand side of (4.1) as $d v(\tau)$ ranges over a set of measures satisfying suitable moment constraints. More precisely, consider the asymptotic expansion for small $P e$ from expression (4.19),

$$
\frac{\kappa^{*}}{\kappa_{0}}=1+\sum_{n=1}^{\infty} P e^{2 n} a_{2 n}
$$

with

$$
a_{2 n}=\frac{(-1)^{n-1}}{d} \int_{-\infty}^{+\infty} \tau^{2(n-1)} v(d \tau),
$$

$n=1,2,3, \ldots$. Recalling that the moments of $v(d \tau)$ correspond to multiple integrals obtained by formal perturbation theory,

$$
a_{2 n}=\frac{1}{d} \operatorname{Tr}\left\langle\mathbf{H} \cdot(\boldsymbol{\Gamma} \cdot \mathbf{H})^{2 n-1}\right\rangle,
$$

- which can be computed or suitably estimated in principle - let us assume that the numerical values $a_{2}, a_{4}, \ldots, a_{2 N}$ in (4.3) are known. Then, upper and lower bounds on $\kappa^{*} / \kappa_{0}$ can be derived by varying $d v$ in (4.1) over all probability measures satisfying the moment constraints (4.4) for $n \leqq N$. This leads to the bounds

$$
\frac{\kappa^{*}}{\kappa_{0}} \leqq 1+\max _{\tilde{v}} \frac{1}{d} \int_{-\infty}^{+\infty} \frac{P e^{2} d \tilde{v}(\tau)}{1+P e^{2} \tau^{2}}
$$

and

$$
\frac{\kappa^{*}}{\kappa_{0}} \geqq 1+\min _{\tilde{v}} \frac{1}{d} \int_{-\infty}^{+\infty} \frac{P e^{2} d \tilde{v}(\tau)}{1+P e^{2} \tau^{2}},
$$

where $\tilde{v}$ ranges over the set of all probability measures satisfying

$$
\frac{1}{d} \int_{-\infty}^{+\infty} \tau^{2(n-1)} \tilde{v}(d \tau)=(-1)^{n+1} a_{2 n}, \quad n \leqq N .
$$

Wheeler and Gordon [32] have shown that the extremal values in (4.6), (4.7) are achieved by measures consisting of finite convex combinations of Dirac masses, and that the corresponding bounds which are functions of $P e^{2}$ are suitable Padé approximants for (4.1).

Recall that the Padé approximant $[m, n](z)$ of a function $f(z)$ is defined as

$$
[m, n](z) \equiv \frac{P_{m}(z)}{P_{n}(z)},
$$

where $P_{m}(z), P_{n}(z)$ are respectively polynomials of degree $m$ an $n$, determined by 
the constraints

$$
\left[\left(\frac{d}{d z}\right)^{r} \frac{P_{m}(z)}{P_{n}(z)}\right]_{z=0}=\left.\left(\frac{d}{d z}\right)^{r} f(z)\right|_{z=0} \quad 0 \leqq r \leqq m+n+2 .
$$

According to Wheeler and Gordon [32], the approximants [n, $n-1]$ and $[n, n]$ have the following extremal properties:

$$
[n, n-1]\left(P e^{2}\right)=1+\max _{\tilde{v}} \frac{1}{d} \int_{-\infty}^{+\infty} \frac{P e^{2} d \tilde{v}(\tau)}{1+P e^{2} \tau^{2}},
$$

where $\tilde{v}$ satisfies (4.8) with $N=2 n-1$;

$$
[n, n]\left(P e^{2}\right)=1+\min _{\tilde{v}} \frac{1}{d} \int_{-\infty}^{+\infty} \frac{P e^{2} d \tilde{v}(\tau)}{1+P e^{2} \tau^{2}},
$$

where $\tilde{v}$ satisfies (4.8) with $N=2 n$.

We therefore conclude, from (4.6), (4.7), that the following bounds hold for all $n \geqq 1$ :

$$
\kappa_{0}[n, n]\left(P e^{2}\right) \leqq \kappa^{*} \leqq \kappa_{0}[n, n-1]\left(P e^{2}\right)
$$

From general principles on the approximation of Stieltjes' integrals, the bounds (4.13) are known to converge to the effective conductivity as $n \rightarrow \infty$; i.e., as more and more statistical information (in the form (4.5)) is incorporated into the bounds. A few approximants, for low, $n$, can be readily computed from (4.10). We have

$$
\begin{gathered}
{[1,0]\left(P e^{2}\right)=1+\frac{1}{d} P e^{2},} \\
{[1,1]\left(P e^{2}\right)=1+\frac{1}{d} \frac{P e^{2}}{1+d\left|a_{4}\right| P e^{2}},} \\
{[2,1]\left(P e^{2}\right)=1+\frac{1}{d} P e^{2}+\frac{a_{4}^{2} P e^{4}}{a_{4}-a_{6} P e^{2}},}
\end{gathered}
$$

and

where

$$
[2,2]\left(P e^{2}\right)=\frac{1+\alpha_{1} P e^{2}+\alpha_{2} P e^{4}}{1+\beta_{1} P e^{2}+\beta_{2} P e^{4}},
$$

$$
\begin{array}{ll}
\beta_{1}=\frac{a_{2} a_{8}-a_{4} a_{6}}{a_{4}^{2}-a_{2} a_{6}}, & \beta_{2}=\frac{a_{6}^{2}-a_{4} a_{8}}{a_{4}^{2}-a_{2} a_{6}}, \\
\alpha_{1}=a_{2}+\beta_{1} \text { and } & \alpha_{2}=a_{4}+a_{2} \beta_{1}+\beta_{2} .
\end{array}
$$

The Stieltjes integral representation can also be useful to determine, by extrapolation, bounds on the effective diffusivity $\kappa^{*}$, given that its exact or approximate value is known for one or more values of $P e$ and $\kappa_{0}$. Such an approach may be useful for checking the validity of Monte Carlo simulations for the effective diffusivity. 
IV.2. Realizability of $\kappa^{*}=\kappa_{0}[1,0]\left(P e^{2}\right)$ for Three Dimensional Flows. Let $\mathbf{u}$ be a simple shear flow:

$$
\mathbf{u}(x)=\left(\begin{array}{c}
0 \\
0 \\
u\left(x_{1}\right)
\end{array}\right)
$$

so that Eq. (1) becomes

$$
\frac{\partial T(x, t)}{\partial t}+u\left(x_{1}\right) \frac{\partial T}{\partial x_{3}}(x, t)=\kappa_{0} \Delta T(x, t) .
$$

The field $\mathbf{H}_{1}(x)$ is given explicitly by

$$
\mathbf{H}_{1}(x)=\left[\begin{array}{ccc}
0 & 0 & \phi_{1}\left(x_{1}\right) \\
0 & 0 & 0 \\
-\phi\left(x_{1}\right) & 0 & 0
\end{array}\right] \equiv \mathbf{J}_{13} \phi_{1}\left(x_{1}\right)
$$

where

$$
\phi_{1}\left(x_{1}\right)=\int_{-\infty}^{+\infty} e^{i k_{1} x_{1}} \frac{d \hat{u}\left(k_{1}\right)}{i k_{1}}
$$

is the stream function for the flow (4.18). We nondimensionalize the problem by setting

$$
P e^{2}=\frac{\left\langle\phi_{1}^{2}\right\rangle}{\kappa_{0}^{2}}=\frac{1}{\kappa_{0}^{2}} \int_{-\infty}^{+\infty} \frac{\left\langle\left|d \hat{u}\left(k_{1}\right)\right|^{2}\right\rangle}{k_{1}^{2}}
$$

and defining the nondimensional velocity potential $\phi$ by

$$
\phi_{1}\left(x_{1}\right)=\kappa_{0} \operatorname{Pe} \phi\left(x_{1}\right)
$$

Note in particular that $\left\langle\phi^{2}\right\rangle=1$. The field $\mathbf{E}=\mathbf{E}\left(x_{1}\right)$ satisfying the basic equations

is given by

$$
\begin{aligned}
& \nabla \cdot(\mathbf{I}+P e \mathbf{H}) \cdot \mathbf{E}=0, \\
& \nabla \times \mathbf{E}=0, \quad\langle\mathbf{E}\rangle=\mathbf{I},
\end{aligned}
$$

$$
\mathbf{E}\left(x_{1}\right)=\left[\begin{array}{ccc}
1 & 0 & \operatorname{Pe} \phi\left(x_{1}\right) \\
0 & 1 & 0 \\
0 & 0 & 1
\end{array}\right] \text {, }
$$

so that, by the arguments of Sect. 3 , 


$$
\begin{aligned}
\stackrel{\kappa}{*}^{*} & =\kappa_{0}\left\langle\mathbf{E}^{T} \cdot \mathbf{E}\right\rangle \\
& =\kappa_{0}\left[\begin{array}{ccc}
1 & 0 & 2 P e\langle\phi\rangle \\
0 & 1 & 0 \\
2 P e\langle\phi\rangle & 0 & 1+P e^{2}\left\langle\phi^{2}\right\rangle
\end{array}\right] \\
& =\kappa_{0}\left[\begin{array}{ccc}
1 & 0 & 0 \\
0 & 1 & 0 \\
0 & 0 & 1+P e^{2}
\end{array}\right] .
\end{aligned}
$$

Observe that (4.25) corresponds to the anisotropic representation measure

$$
d \underset{\sim}{\mu}(\tau)=\left[\begin{array}{ccc}
0 & 0 & 0 \\
0 & 0 & 0 \\
0 & 0 & \delta(\tau) d \tau
\end{array}\right]=\delta(\tau) \mathbf{e}_{3} \otimes \mathbf{e}_{3},
$$

where $\mathbf{e}_{3}$ represents a unit vector along the direction $x_{3}$. Taking the trace of (4.25), we obtain

$$
\frac{1}{3} \operatorname{Tr} \underset{\sim}{*}{ }^{*}=\kappa_{0}\left[1+\frac{1}{3} P e^{2}\right]
$$

which is the upper bound corresponding to the approximant $[1,0]$. It is not immediately clear, however, that the upper bound can be realized by isotropic flow configurations (for which $\kappa_{\sim}^{*}=\kappa^{*} \mathbf{I}$ ). We argue here that this is indeed true, i.e., that values of $\kappa^{*}$ arbitrarily close to the right-hand side of (4.27) can indeed be attained by suitable isotropic flows.

The construction of such flows is done using an argument involving separation of scales introduced by K. Schulgasser in the study of composite materials [33], and subsequently implemented by other authors [34, 35]. Since the effective diffusivity of a simple shear flow is a uniaxial tensor with eigenvalues $\kappa_{0}$ (double) and $\kappa_{0}\left(1+P e^{2}\right)$ (simple), we view such flow, on a macroscopic scale, as a homogeneous, anisotropic diffusive medium, e.g. a heat conductor, and seek a geometric configuration, in "polycrystalline" form, of this anisotropic medium that gives rise to an isotropic effective diffusivity

$$
\kappa^{*}=\frac{1}{3}\left[\kappa_{0}+\kappa_{0}+\kappa_{0}\left(1+P e^{2}\right)\right]=\kappa_{0}\left(1+\frac{1}{3} P e^{2}\right)
$$

which is the arithmetic mean of the three principal diffusivities. To construct such a macroscopically isotropic polycrystal, one proceeds in two steps. First, we layer periodically the "pure crystals"

$$
\left[\begin{array}{ccc}
\kappa_{0} & 0 & 0 \\
0 & \kappa_{0} & 0 \\
0 & 0 & \kappa_{0}\left(1+P e^{2}\right)
\end{array}\right] \text { and }\left[\begin{array}{ccc}
\kappa_{0} & 0 & 0 \\
0 & \kappa_{0}\left(1+P e^{2}\right) & 0 \\
0 & 0 & \kappa_{0}
\end{array}\right]
$$

in parallel slabs (laminates) of thicknessess $\frac{2}{3} l_{1}$ and $\frac{1}{3} l_{1}$, respectively, where $l_{1}$ is a given length, the slabs being perpendicular to the unit vector $\mathbf{e}_{1}$ in the direction $x_{1}$. The effective diffusivity tensor for this configuration is $[33,34]$ 


$$
\stackrel{\sim}{\kappa}^{*}=\left[\begin{array}{ccc}
\kappa_{0} & 0 & 0 \\
0 & \kappa_{0}\left(1+\frac{2}{3} P e^{2}\right) & 0 \\
0 & 0 & \kappa_{0}\left(1+\frac{1}{3} P e^{2}\right)
\end{array}\right] \text {. }
$$

In a second step, we layer the tensor ${\underset{\sim}{1}}_{1}^{*}$ with itself after a rotation of $90^{\circ}$ about the $x_{3}$-axis, i.e., with the tensor

$$
{\underset{\sim}{2}}_{2}^{*}=\left[\begin{array}{ccc}
\kappa_{0}\left(1+\frac{2}{3} P e^{2}\right) & 0 & 0 \\
0 & \kappa_{0} & 0 \\
0 & 0 & \kappa_{0}\left(1+\frac{1}{3} P e^{2}\right)
\end{array}\right]
$$

in layers of thickness $l_{2}$ for both crystals, perpendicularly to the unit vector $\mathbf{e}_{3}$. The length $l_{2}$ is chosen so that $l_{1} \ll l_{2}$. The corresponding effective diffusivity tensor is obtained by averaging the eigenvalues of (4.30) and (4.31) in the directions $\mathbf{e}_{1}$ and $\mathbf{e}_{2}=\left[\begin{array}{l}0 \\ 1 \\ 0\end{array}\right]$, so that, finally,

$$
\kappa_{3}^{*}=\kappa_{0}\left(1+\frac{1}{3} P e^{2}\right) \mathbf{I}
$$

To relate this construction to our flow problem, we choose $l_{1}$ very large compared to the typical wavelength of the function $u\left(x_{1}\right)$ of the simple shear flow (4.18). The configuration consisting of parallel slabs of crystals (4.29) has approximately the same effective diffusivity as a periodic flow with period $l_{1}$, $\mathbf{u}(x)=\mathbf{u}\left(x_{1}\right)$, such that

$$
\mathbf{u}_{\varepsilon}\left(x_{1}\right)= \begin{cases}u\left(x_{1}\right) \mathbf{e}_{3} & \varepsilon l_{1} \leqq x_{3} \leqq \frac{2}{3} l_{1}-\varepsilon l_{1} \\ u\left(x_{1}\right) \mathbf{e}_{2} & \frac{2}{3} l_{1}+\varepsilon l_{1}<x_{3}<l_{1}-\varepsilon l_{1},\end{cases}
$$

where $\varepsilon \ll 1$, and $2 \varepsilon$ represents the thickness of a very thin transition layer along which $\mathbf{u}\left(x_{1}\right)$ rotates continuously by $90^{\circ}$ through the $x_{1}$-axis. Since the normal component of $\mathbf{u}(x)$ near the transition is equal to zero, the vector field $\mathbf{u}_{\varepsilon}$ can be taken to be incompressible. (In the limit $\varepsilon \rightarrow 0$, this transition layer can be interpreted as a vortex sheet ). At the second level of this hierarchical construction, corresponding to the layering of (4.30) and (4.31), we interpret this layering as a periodic flow with period $2 l_{2}$ in the $x_{3}$ direction, obtained by juxtaposing $\mathbf{u}_{\varepsilon}$ and a flow consisting of a rotation of $\mathbf{u}_{\varepsilon}$ by $90^{\circ}$ degrees, connected by a transition layer of thickness $\varepsilon l_{2}$. The argument of iterated homogenization presented here implies that such incompressible flows have an effective diffusivity tensor $\underset{\sim}{\kappa}, l_{1}, 1_{2}$ such that

$$
\lim _{\varepsilon \downarrow 0} \lim _{l_{1} / l_{2} \rightarrow 0}{\underset{\sim}{\kappa}, l_{1}, l_{2}}^{*}=\kappa_{0}\left(1+\frac{1}{3} P e^{2}\right) \mathbf{I}
$$

The discussion of multiple-scale homogenization and its relation to various effective medium theories is given in Avellaneda [36]. Such work provides a set of formal mathematical tools for making the above arguments fully rigorous.

We hasten to observe that the flows just described are unphysical and have probably litte or no relation to actual hydrodynamic flows. They serve, however, the important purpose of showing that for three-dimensional flows, improvements of the upper bound 


$$
\kappa^{*} \leqq \kappa_{0}\left(1+\frac{1}{3} P e^{2}\right),
$$

should necessarily incorporate other statistical or dynamical information about the Eulerian velocity field $\mathbf{u}$ such as the $2 n$-point correlation functions, for $n \geqq 2$. Moreover, this realizability result provides us with an approximate idea of what type of flows satisfy approximately the bound $\kappa *=\kappa_{0}[1,0]\left(P e^{2}\right)$.

IV.3. Realizability of $\kappa^{*}=\kappa_{0}[1,1]\left(P e^{2}\right)$ for Three-Dimensional Flows. Pursuing further the analogy with the theory of composite materials, we construct another yet highly idealized class of flows that saturates (i.e., reaches asymptotically) the lower bound

$$
\kappa^{*} \geqq \kappa_{0}\left[1+\frac{1}{3} \frac{P e^{2}}{1-3 a_{4} P e^{2}}\right]
$$

This construction is based on the coated-sphere model, introducted by Hashin and Shtrikman as model of a two-phase composite with explicitly computable effective diffusivity. We will consider a flow of the type constructed in the previous section, with $\varepsilon \ll 1$, and $l_{1} \ll l_{2}$, so that the value of the corresponding diffusivity ${\underset{\sim}{\varepsilon, l_{1}, l_{2}}}_{*}$ is arbitrarily close to $\kappa^{*}=\kappa_{0}\left(1+\frac{1}{3} P e^{2}\right)$. We treat such a flow macroscopically as an isotropic diffusive medium with (scalar) diffusivity ${\underset{\sim}{\varepsilon}, l_{1}, l_{2}}_{*}$. Following Hashin and Shtrikman [25], we consider a space-filling configuration of composite spheres, consisting of a core of diffusivity $\underset{\sim}{\underset{\varepsilon}{*}, l_{1}, l_{2}}$, surrounded by a shell of diffusivity $\kappa_{0}$. Note that the radii of the composite spheres must range down to infinitesimal sizes, so as to form a space-filling configuration. The ratio of the core radius to the overall radius is the same for all the coated spheres. Therefore, the volume fraction occupied by the "material" with diffusivity ${\stackrel{\kappa}{*}, l_{1}, l_{2}}_{\text {is }}^{*}$ isual for all spheres, say equal to $p, 0<p<1$. An exact, self-consistent calculation of the overall effective diffusivity, based on the classical dipole expansion of the potential around a composite sphere embedded in an infinite homogeneous medium yields the value [25]

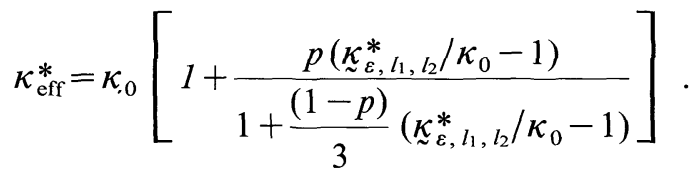

Thus, from (4.34),

$$
\lim _{\varepsilon \downarrow 0} \lim _{l_{1} / l_{2} \rightarrow 0} \kappa_{\text {eff }}^{*}=\kappa_{0}\left[1+\frac{1}{3} \frac{p P e^{2}}{1+\frac{(1-p)}{9} P e^{2}}\right] .
$$

We relate the configuration just described to a flow field, using ideas of separation of scales of [36] by considering the $\mathbf{H}_{1}$-matrix field corresponding to the multiply layered flow of IV.2, realizing the Padé $[1,0]$ bound. The Péclet number of such a flow is, by definition, given by

$$
\begin{aligned}
P e^{2} & =\frac{1}{\kappa_{1}^{2}} \operatorname{tr}\left\langle\mathbf{H}_{1} \boldsymbol{\Gamma} \mathbf{H}_{1}\right\rangle . \\
& =\frac{1}{2 \kappa_{0}^{2}} \operatorname{tr}\left\langle\mathbf{H}_{1}^{T} \mathbf{H}_{1}\right\rangle .
\end{aligned}
$$


Consider now the matrix $\widetilde{\mathbf{H}}_{1, \theta}(x)$ defined by

$$
\tilde{\mathbf{H}}_{1, \theta} \equiv \sum_{\alpha} \mathbf{H}_{1}\left(\frac{x}{\theta}\right) 1_{C_{\alpha}}(x),
$$

where $\theta \ll 1$ is a small parameter and $1_{C_{\alpha}}$ is the characteristic function of the core $C_{\alpha}$ of the composite sphere $B_{\alpha}$ in the aggregate. Thus the core of each composite sphere is replaced by a rapidly varying flow with $\mathbf{H}$-field equal to $\mathbf{H}_{1}(x / \theta)$. This field is not smooth at the boundary of the cores and hence gives rise to spherical vortex sheets around each core. If necessary, a thin transition layer, corresponding to a smoothing of the characteristic functions $\mathbf{1}_{C_{\alpha}}(x)$ can be introduced, to obtain a smooth flow. Since the flow in the core varies much faster than the typical spherical radius, by the principle of separation of scales, or iterated homogenization, introduced in [12] and developed in Avellaneda [36], the effective diffusivity of the flow corresponding to $\tilde{\mathbf{H}}_{1, \theta}(x), \underset{\sim}{\kappa} \kappa_{l_{1}, l_{2}, \theta}$ converges as $\theta \rightarrow 0$, to $\kappa_{\text {eff }}^{*}$, given by (4.37), and hence

$$
\lim _{\varepsilon \downarrow 0} \lim _{l_{1} / l_{2} \rightarrow 0} \lim _{\theta \rightarrow 0}{\underset{\sim}{\kappa}, l_{1}, l_{2}, \theta}^{*}=\kappa_{0}\left[1+\frac{1}{3} \frac{p P e^{2}}{1+\frac{1-p}{9} P e^{2}}\right] \text {. }
$$

Finally, the effective Peclet number of the flow associated to $\mathbf{H}_{1, \theta}$ is given by

$$
\begin{aligned}
\widetilde{P e^{2}} & =\frac{1}{2 \kappa_{0}^{2}} \operatorname{Tr}\left\langle\tilde{\mathbf{H}}_{1, \theta}^{T} \tilde{\mathbf{H}}_{1, \theta}\right\rangle \\
& =\frac{1}{2 \kappa_{0}^{2}} \lim _{R \rightarrow \infty} \frac{1}{8 R^{3}} \int_{\left|x_{i}\right| \leqq R} \operatorname{Tr} \tilde{\mathbf{H}}_{1, \theta}^{T}(x) \tilde{\mathbf{H}}_{1, \theta}(x) d x \\
& =\frac{1}{2 \kappa_{0}^{2}} \lim _{R \rightarrow \infty} \frac{1}{8 R^{3}} \sum_{\cup B_{\alpha} \subseteq Q(R)} \operatorname{Tr} \int_{C_{\alpha}} \tilde{\mathbf{H}}_{1}^{T}\left(\frac{x}{\theta}\right) \mathbf{H}_{1}\left(\frac{x}{\theta}\right) d x,
\end{aligned}
$$

where $Q(R)=\left\{x \in \mathbb{R}^{2}:\left|x_{i}\right| \leqq R\right\}$. Hence

$$
\begin{aligned}
\lim _{\theta \rightarrow 0} \widetilde{P e^{2}} & =\frac{1}{2 \kappa_{0}^{2}} \lim _{R \rightarrow \infty} \frac{1}{8 R^{3}} \sum_{B_{\alpha} \subseteq Q(R)} \lim _{\theta \rightarrow 0} \int_{C_{\alpha}} \operatorname{Tr} \mathbf{H}_{1}^{T}\left(\frac{x}{\theta}\right) \mathbf{H}_{1}\left(\frac{x}{\theta}\right) d x \\
& =\frac{1}{2 \kappa_{0}^{2}} \lim _{R \rightarrow \infty} \frac{1}{8 R^{3}} \sum_{B_{\alpha} \subset Q(R)} \operatorname{Tr}\left\langle\mathbf{H}_{1}^{T} \mathbf{H}_{1}\right\rangle \operatorname{Vol}\left(C_{\alpha}\right) \\
& =\frac{p}{2 \kappa_{0}^{2}} \operatorname{Tr}\left\{\mathbf{H}_{1}^{T} \mathbf{H}_{1}\right\rangle \\
& =p P e^{2},
\end{aligned}
$$

where we used (4.40). Thus, substituting (4.43) ino (4.41) we obtain

$$
\lim \kappa_{\mathrm{eff}}^{*}=\kappa_{0}\left(1+\frac{1}{3} \frac{\widetilde{P e^{2}}}{1+\frac{1-p}{9 p} \widetilde{P e^{2}}}\right)
$$


which is excactly the lower bound corresponding to the $[1,1]$ Padé approximant. Notice that the coefficient $\frac{1-p}{9 p}=t_{p}$ in the denominator of this expression takes all values such that $0 \leqq t_{p}<\infty$. For $p=1$, corresponding to $\tilde{\mathbf{H}}_{1, \theta}(x)=\mathbf{H}_{1}(x / \theta)$, we recover the extremal flow of Sect. IV.2, realizing the upper bound for the [1, 0] Padé approximant. For $0<p<1$, the extremely idealized class of flows just described consists of isolated, high velocity patches, surrounded by an infinite, connected region where the velocity is nearly equal to zero, occupying a volume fraction of space $1-p$. The numerical value of the corresponding effective diffusivity is arbitrarily close to the one predicted by the Padé $[1,1]$ lower bound.

\section{A Variational Principle for the Effective Diffusivity}

In this section we show how $\kappa^{*}$ can be obtained by minimization of a suitable functional defined on curl-free-fields. This variational principle yields an upper bound for the effective diffusivity if one substitutes an appropriate trial field in the functional. The functional that needs to be minimized is non-local, i.e., given a trial field $\mathbf{F}$, it involves not only $\mathbf{F}$ but also the calculation of the values of an integral operator applied to $\mathbf{F}$. The actual computation of the value of this functional for a given field is analogous to solving an electrostatic polarization problem for a system of distributed charges - or finding the hydrodynamic flow field arising from a homogeneous configuration of sources and sinks. Fortunately, in certain cases, such a calculation can be greatly simplified by choosing a suitable trial field $\mathbf{F}$, so that a numerical upper bound for $\kappa^{*}$ can be obtained. We shall illustrate this point here by applying the variational principle to flow fields consisting of arrays of vortices. We note that Krommes and Smith [4] and Kim and Krommes [37] have obtained variational principles for the dissipation rate in diffusion-advection problems which are different from this one.

V.1. The Derivation. We assume first that the field $\mathbf{H}(x)$ under consideration is uniformly bounded, i.e. that $\mathbf{H} \in L^{\infty}(\langle\cdot\rangle)$. This restriction allows us to use a Hilbert space argument for the derivation of the variational principle. The assumption is subsequently removed by an approximation argument.

We define the operator

$$
\mathbf{K}=\mathbf{H} \Gamma \mathbf{H} \text {, }
$$

which is a bounded, linear operator on $L^{2}(\langle\cdot\rangle)$. Moreover, since $\mathbf{H}+\mathbf{H}^{T}=0$, and $\boldsymbol{\Gamma}^{2}=-\boldsymbol{\Gamma}$, we have

$$
\mathbf{K}=-\mathbf{H}^{T} \boldsymbol{\Gamma} \mathbf{H}=\left(\mathbf{H}^{T} \boldsymbol{\Gamma}\right)(\boldsymbol{\Gamma} \mathbf{H})=(\Gamma \mathbf{H})^{T}(\Gamma \mathbf{H}),
$$

so that $\mathbf{K}$ is positive and self-adjoint. We claim that the effective diffusivity for time-independent flows $\mathbf{u}=\mathbf{u}(x)$ with $\mathbf{H} \in L^{\infty}(\langle\cdot\rangle)$ satisfies the following

Variational Principle. For all vectors $\mathbf{e} \in \mathbf{R}^{d}$, we have

$$
\mathbf{e} \cdot{\underset{\sim}{\kappa}}^{*} \cdot \mathbf{e}=\min _{\substack{\mathbf{f} \in L^{2}(\langle\cdot\rangle) \\ \nabla \times \mathbf{f}=0 \\\langle\mathbf{f}\rangle=\mathbf{e}}} \kappa_{0}\left\langle|\mathbf{f}|^{2}+P e^{2} \mathbf{f}^{T} \cdot \mathbf{K} \cdot \mathbf{f}\right\rangle .
$$


Proof. In fact, the Euler-Lagrange equation for this variational problem is the divergence-form equation

$$
\begin{aligned}
\nabla \cdot\left[\left(\mathbf{I}+P e^{2} \mathbf{K}\right) \mathbf{f}\right] & =0, \\
\nabla \times \mathbf{f} & =0, \\
\langle\mathbf{f}\rangle & =\langle\mathbf{e}\rangle .
\end{aligned}
$$

Note however that the "diffusivity" $\mathbf{I}+P e^{2} \mathbf{K}$ is positive, and self-adjoint but non-local, since $\mathbf{K}$ is defined in terms of the integral operator $\mathbf{\Gamma}$. By linearity, we have,

$$
\mathbf{f}=\mathbf{F} \cdot \mathbf{e},
$$

where $\mathbf{F}$ is a matrix-valued function satisfying the equations

$$
\begin{aligned}
\nabla \cdot\left[\left(\mathbf{I}+P e^{2} \mathbf{K}\right) \cdot \mathbf{F}\right] & =0, \\
\nabla \times \mathbf{F} & =0, \quad\langle\mathbf{F}\rangle=\mathbf{I} .
\end{aligned}
$$

Since $\mathbf{F}=\mathbf{I}-\boldsymbol{\Gamma} \mathbf{F}$, and $-\boldsymbol{\Gamma}$ is the projection operator onto the subspace $V$ of $L^{2}(\langle\cdot\rangle)$ defined by

$$
V=\left\{\mathbf{F} \in L^{2}(\langle\cdot\rangle) \mid \nabla \times \mathbf{F}=0 ; \quad\langle\mathbf{F}\rangle=0\right\},
$$

Eqs. (5.6) can be rewritten as the integral equation

$$
\mathbf{F}-P e^{2} \boldsymbol{\Gamma} \mathbf{K F}=\mathbf{I} .
$$

Separating $\mathbf{F}$ into fluctuating and average components, we obtain $\mathbf{F}=\mathbf{F}^{\prime}+\mathbf{I}$ with $\mathbf{F}^{\prime}$ satisfying

$$
\mathbf{F}^{\prime}-P e^{2} \boldsymbol{\Gamma} \mathbf{K F}^{\prime}=P e^{2} \boldsymbol{\Gamma} \mathbf{K I}, \quad \mathbf{F}^{\prime} \in V .
$$

We proceed as in Sect. III, observing that the operator

$$
\mathrm{S} \equiv \boldsymbol{\Gamma K}
$$

is negative and self-adjoint on $V$. Consequently, introducing the resolution of the identity $\mathbf{U}(\theta)$ of the operator $\mathbf{S}$, we can write

$$
\mathbf{F}^{\prime}=\int_{-\infty}^{0} \frac{P e^{2} d \mathbf{U}(\theta) \boldsymbol{\Gamma} \mathbf{K I}}{1-P e^{2} \theta}=\int_{0}^{\infty} \frac{P e^{2} d \mathbf{U}(-\theta) \Gamma \mathbf{K} \mathbf{I}}{1+P e^{2} \theta} .
$$

Hence, we have

$$
\left\langle\mathbf{I K F ^ { \prime }}\right\rangle=\int_{0}^{\infty} \frac{P e^{2} d \mathbf{m}(\theta)}{1+P e^{2} \theta^{2}},
$$

where

$$
\mathbf{m}(\theta)=\langle\mathbf{I K U}(-\theta) \boldsymbol{\Gamma} \mathbf{K I}\rangle,
$$


and, consequently,

$$
\begin{aligned}
\underset{\mathbf{f}}{\min }\left\langle|\mathbf{f}|^{2}+P e^{2} \mathbf{f}^{T} \cdot \mathbf{K} \cdot \mathbf{f}\right\rangle & =\mathbf{e}\left\langle\left(\mathbf{I}+P e^{2} \mathbf{K}\right) \cdot \mathbf{F}\right\rangle \mathbf{e} \\
& =\mathbf{e}\left(\mathbf{I}+P e^{2}\langle\mathbf{K}\rangle+P e^{2}\left\langle\mathbf{K} \mathbf{F}^{\prime}\right\rangle\right) \mathbf{e} \\
& =\mathbf{e} \cdot\left(\mathbf{I}+P e^{2}\langle\mathbf{K}\rangle+\int_{0}^{\infty} \frac{P e^{4} d \mathbf{m}(\theta)}{1+P e^{2} \theta}\right) \cdot \mathbf{e} \\
& =\mathbf{e} \cdot\left(\mathbf{I}+P e^{2}\langle\mathbf{H} \boldsymbol{\Gamma} \mathbf{H}\rangle+\int_{0}^{\infty} \frac{P e^{4} d \mathbf{m}(\theta)}{1+P e^{2} \theta}\right) \cdot \mathbf{e} .
\end{aligned}
$$

The successive moments of the measure $d \mathbf{m}(\theta)$ are determined from (5.13); we have,

$$
\begin{aligned}
\int_{0}^{\infty} \theta^{n} d \mathbf{m}(\theta) & =(-1)^{n} \int_{0}^{\infty} \theta^{n} d\langle\mathbf{I K U} \mathbf{U}(\theta) \boldsymbol{\Gamma} \mathbf{K} \mathbf{I}\rangle \\
& =(-1)^{n}\left\{\mathbf{K}(\boldsymbol{\Gamma} \mathbf{K})^{n} \boldsymbol{\Gamma} \mathbf{K}\right\rangle=(-1)^{n}\left\langle\mathbf{H}(\boldsymbol{\Gamma} \mathbf{H})^{2 n+3}\right\rangle .
\end{aligned}
$$

In particular,

$$
\begin{aligned}
& \int_{0}^{\infty} d \mathbf{m}(\theta)=\left\langle\mathbf{H}(\boldsymbol{\Gamma} \mathbf{H})^{3}\right\rangle, \\
& \int_{0}^{\infty} \theta d \mathbf{m}(\theta)=-\left\langle\mathbf{H}(\boldsymbol{\Gamma} \mathbf{H})^{5}\right\rangle, \quad \text { etc. }
\end{aligned}
$$

It follows that the measures $d \mathbf{m}$ and $\underset{\sim}{\mu}$ are related by the formula

$$
d \mathbf{m}\left(\tau^{2}\right)=-\tau^{2} d \mu(\tau), \quad-\infty<\tau<\infty,
$$

so that, as claimed,

$$
\begin{aligned}
\min _{\mathbf{f}} \kappa_{0}\left\langle|\mathbf{f}|^{2}+P e^{2} \mathbf{f}^{T} \cdot \mathbf{K} \cdot \mathbf{f}\right\rangle= & \kappa_{0} \mathbf{e} \cdot\left(\mathbf{I}+P e^{2} \int_{-\infty}^{+\infty} d \underset{\sim}{\mu}\right. \\
& \left.-\int_{-\infty}^{+\infty} \frac{\tau^{2} P e^{4} d \underset{\sim}{\mu}(\tau)}{1+\tau^{2} P e^{2}}\right) \cdot \mathbf{e} \\
= & \kappa_{0} \mathbf{e} \cdot\left(\mathbf{I}+\int_{-\infty}^{+\infty} \frac{P e^{2} d \mu(\tau)}{1+\tau^{2} P e^{2}}\right) \cdot \mathbf{e} \\
& =\mathbf{e} \cdot{\underset{\sim}{\kappa}}^{*} \cdot \mathbf{e} .
\end{aligned}
$$

This variational principle can be extended to unbounded $\mathbf{H}$-fields, satisfying the weaker condition $\mathbf{H} \in L^{4}(\langle\cdot\rangle)$. In fact, let $V_{4}$ be the space

$$
V_{4}=\left\{\mathbf{f} \in L^{4}(\langle\cdot\rangle): \nabla \times \mathbf{f}=0\right\} \text {. }
$$


The corresponding variational principle for $\kappa_{\sim}^{*}$ is

$$
\mathbf{e}_{T} \cdot{\underset{\sim}{\kappa}}^{*} \cdot \mathbf{e}=\min _{\substack{\mathbf{f} \in V_{4} \\\langle\mathbf{f}\rangle=\mathbf{e}}} \kappa_{0}\left\langle|\mathbf{f}|^{2}+P e^{2}|\boldsymbol{\Gamma} \mathbf{H} \mathbf{f}|^{2}\right\rangle .
$$

To see this, consider the truncated $\mathbf{H}$-fields, $\mathbf{H}^{(n)}, n=1,2,3, \ldots$, and the corresponding solutions to the basic equations, $\mathbf{E}^{(n)}, n=1,2,3, \ldots$. Since $\mathbf{H}^{(n)} \in L^{\infty}(\langle\cdot\rangle)$, we have a variational principle for the diffusivity corresponding to the field $\mathbf{H}^{(n)}$, namely,

$$
\begin{aligned}
\mathbf{e}^{T} \cdot{\underset{\sim}{n}}_{n}^{*} \cdot \mathbf{e} & \equiv \kappa_{0}\left[|\mathbf{e}|^{2}+\left\langle\left|\mathbf{E}^{(n)} \mathbf{e}\right|^{2}\right\rangle\right] \\
& \leqq \kappa_{0}\left\langle|\mathbf{f}|^{2}+P e^{2}\left|\boldsymbol{\Gamma} \mathbf{H}^{(n)} \mathbf{f}\right|^{2}\right\rangle,
\end{aligned}
$$

for all $\mathbf{f} \in L^{4}(\langle\cdot\rangle)$. Letting $n \rightarrow \infty$ on both sides of this inequality, and subsequently minimizing the right-hand side over $f \in L^{4}(\langle\cdot\rangle)$, we obtain

$$
\mathbf{e}^{T} \cdot{\underset{\sim}{\kappa}}^{*} \cdot \mathbf{e} \leqq \min _{\substack{\mathbf{f} \in V_{4} \\\langle\mathbf{f}\rangle=\mathbf{e}}} \kappa_{0}\left\langle|\mathbf{f}|^{2}+P e^{2}|\boldsymbol{\Gamma} \mathbf{H} \mathbf{f}|^{2}\right\rangle .
$$

To obtain the opposite inequality to (5.22), we denote by $f^{*}$ the minimizer of the right-hand side of (5.22). Then, since $\Gamma$ is a bounded operator in $L^{2}(\langle\cdot\rangle)$ and $\mathbf{H}^{(n)} \mathbf{f}^{*} \rightarrow \mathbf{H} \mathbf{f}^{*}$ in $L^{2}(\langle\cdot\rangle)$ as $n \rightarrow \infty$, we conclude that for all $\varepsilon>0$, there exists $n_{0}=n_{0}(\varepsilon)$ such that, for all $n \geqq n_{0}$,

$$
\begin{aligned}
\min _{\substack{\mathbf{f} \in V_{4} \\
\langle\mathbf{f}\rangle=\mathbf{e}}} \kappa_{0}\left\langle|\mathbf{f}|^{2}+P e^{2}|\boldsymbol{\Gamma} \mathbf{H} \mathbf{f}|^{2}\right\rangle & \geqq \kappa_{0}\left\langle\left|\mathbf{f}^{*}\right|^{2}+P e^{2}\left|\boldsymbol{\Gamma} \mathbf{H}^{(n)} \mathbf{f}^{*}\right|^{2}\right\rangle-\varepsilon \\
& \geqq \min _{\mathbf{f} \in V} \kappa_{0}\left\langle|\mathbf{f}|^{2}+P e^{2}\left|\boldsymbol{\Gamma} \mathbf{H}^{(n)} \mathbf{f}\right|^{2}\right\rangle-\varepsilon \\
& =\mathbf{e}^{T} \cdot \sim_{n}^{*} \cdot \mathbf{e}-\varepsilon .
\end{aligned}
$$

Letting $n \rightarrow \infty$, we obtain

$$
\min _{\substack{\mathbf{f} \in V_{4} \\\langle\mathbf{f}\rangle=\mathbf{e}}} \kappa_{0}\left\langle|\mathbf{f}|^{2}+P e^{2}|\boldsymbol{\Gamma} \mathbf{H} \mathbf{f}|^{2}\right\rangle \geqq \mathbf{e}^{T} \cdot{\underset{\sim}{\kappa}}^{*} \cdot \mathbf{e}-\varepsilon,
$$

for all $\varepsilon>0$, and hence

$$
\min _{\substack{\mathbf{f} \in V_{4} \\\langle\mathbf{f}\rangle=\mathbf{e}}} \kappa_{0}\left\langle|\mathbf{f}|^{2}+P e^{2}|\boldsymbol{\Gamma} \mathbf{H} \mathbf{f}|^{2}\right\rangle \geqq \mathbf{e}^{T} \cdot \kappa_{\sim}^{*} \cdot \mathbf{e} .
$$

Combining (5.22) and (5.25) we conclude the validity of the extended form of the variational principle (5.20).

V.2. Application. We present a simple application of this variational principle for estimating the diffusivity of certain flows formed by superpositions of vortices. Let $\mathbf{H}^{(0)}(x)$ be a smooth, skew-symmetric matrix-valued function of compact support in $\mathbb{R}^{d}(d=2,3)$, and let $\left\{\xi_{\alpha}\right\}$ be a periodic array of points in $\mathbb{R}^{d}$. We define the periodic $\mathbf{H}$-field,

$$
\mathbf{H}^{(1)}(\mathbf{x})=\sum_{\alpha} \mathbf{H}^{(0)}\left(\mathbf{x}-\xi_{\alpha}\right) .
$$


We assume that the points $\xi_{\alpha}$ are spaced sufficiently far apart so that for $\alpha \neq \beta$ the supports of the functions $\mathbf{H}^{(0)}\left(\mathbf{x}-\xi_{\alpha}\right)$ and $\mathbf{H}^{(0)}\left(\mathbf{x}-\xi_{\beta}\right)$ are separated by a positive distance from each other. Equation (5.26) defines a stationary process in $\mathbb{R}^{d}$. Taking the divergence of both sides of this equation, we obtain a periodic incompressible velocity field $\mathbf{u}(x)$, given by

$$
\mathbf{u}(x)=\sum_{\alpha} \mathbf{u}^{(0)}\left(\mathbf{x}-\xi_{\alpha}\right), \quad \text { with } \quad \mathbf{u}^{(0)}=\nabla \cdot \mathbf{H}^{(0)} .
$$

The Péclet number of the advection-diffusion equation with diffusivity $\kappa_{0}$, for this flow is

$$
\begin{aligned}
P e^{2} & =\frac{1}{\kappa_{0}^{2}} \operatorname{Trace}\left\langle\mathbf{H}^{(1)} \boldsymbol{\Gamma} \mathbf{H}^{(1)}\right\rangle \\
& =\frac{1}{2 \kappa_{0}^{2}} \operatorname{Trace}\left\langle\mathbf{H}^{(1)^{\prime} T} \cdot \mathbf{H}^{(1)^{\prime}}\right\rangle \\
& =\frac{\rho^{2}}{2 \kappa_{0}^{2}}\left[\operatorname{Tr} \int \mathbf{H}^{(0) T}(x) \mathbf{H}^{(0)}(x) d x-\operatorname{Tr}\left(\int \mathbf{H}^{0}(x) d x\right)^{T}\left(\int \mathbf{H}^{0}(x) d x\right)\right],
\end{aligned}
$$

where $\mathbf{H}^{(1)^{\prime}}(x)$ is the fluctuating par of $\mathbf{H}^{(1)}(x)$ and $\rho$ is the volume fraction occupied by the union of all the supports of $\mathbf{H}^{(0)}\left(x-\xi_{\alpha}\right)$ as $\alpha$ varies. We denote this set by $\Sigma$.

The variational principle can be applied to this problem. In fact, for all vectors $\mathbf{e}$ in $\mathbb{R}^{d}$,

$$
\begin{aligned}
\frac{\mathbf{e}^{T} \cdot \kappa^{*} \cdot \mathbf{e}}{\kappa_{0}} & =\inf _{\substack{\mathbf{f} \in V \\
\langle\mathbf{f}\rangle=\mathbf{e}}}\left\langle|\mathbf{f}|^{2}+P e^{2}|\boldsymbol{\Gamma} \mathbf{H} \mathbf{f}|^{2}\right\rangle \\
& \leqq \inf \left\{\left\langle|\mathbf{f}|^{2}\right\rangle, \quad \nabla \times \mathbf{f}=0, \quad \mathbf{f}=0 \text { on } \Sigma, \quad\langle\mathbf{f}\rangle=\mathbf{e}\right\} .
\end{aligned}
$$

The last expression can again be related to a problem in composite materials. In fact, consider a composite consisting of a metallic (conducting) phase contained in $\Sigma$ with dielectric constant $\varepsilon_{m}=\infty$, and a surrounding homogeneous dielectric (insulator), contained in $\mathbb{R}^{d}-\Sigma$ with dielectric constant $\varepsilon_{i}$. The right-hand side of (5.29) coincides with the ratio $\varepsilon_{\text {eff }} / \varepsilon_{i}$, where $\varepsilon_{\text {eff }}$ is the effective dielectric constant. This ratio is clearly finite if the "metallic" phase is disconnected, i.e., if the supports of the functions $\mathbf{H}^{(0)}\left(\mathbf{x}-\xi_{\alpha}\right)$ do not overlap. We conclude that the effective diffusivity of the flow (5.27) satisfies

$$
\kappa^{*} \leqq \kappa_{0} C(\rho),
$$

where $C(\rho)$ is a constant depending on the geometry of the array of vortices. In particular, $\kappa^{*} \propto \kappa_{0}$ for all values of $\kappa_{0}$.

This result applies not only to periodic arrays of vortices, but also to random arrays in which the vortices are allowed to overlap. For instance, we consider a random or Poisson process of points $\left\{\xi_{\alpha}\right\}$ in $\mathbb{R}^{d}$ with intensity $\lambda$, and the velocity field $\mathbf{u}(x)$ defined by (5.26), (5.27). The corresponding support set $\Sigma$, which is now random, is known from classical geometrical percolation theory (see Deutscher et al. [38] or Kesten [39]) to have two distinct phases corresponding respectively to low and high values of the intensity $\lambda$ (=density of points $\xi_{\alpha}$ ). Accordingly, there exists a critical value $\lambda_{c}$ such that we have 
(i) a non-percolating phase: if $\lambda<\lambda_{c}$ then $\Sigma$ is composed of a disjoint union of clusters of vortices having finite diameter, with probability one; and

(ii) a percolating phase: if $\lambda>\lambda_{c}$ then $\Sigma$ contains at least one infinite cluster of vortices with unit probability.

The variational principle (5.20) applies once again to yield, for $\lambda<\lambda_{c}$,

$$
\kappa_{0} \leqq \kappa^{*} \leqq \kappa C(\lambda)
$$

where $C(\lambda)$ is a constant depending on the shape of the support of $\mathbf{H}^{(0)}$ and on the intensity $\lambda$ of the point process. Thus, the variational principle gives an easy proof of the fact that the long time/large scale diffusivity of a random array of vortices below the percolation threshold is, in a sense, trivially renormalized $\left(\kappa^{*} \sim \kappa_{0}\right)$. We expect that the variational principle may be useful to construct accurate upper bounds on $\kappa^{*}$, for situations in which the vortices interact more substantially by using suitable trial fields $\mathbf{f}$. The interesting problem of studying the effective diffusivity for $\lambda>\lambda_{c}$ is currently being investigated numerically [45].

\section{A Special Class of Two-Dimensional Flows}

This section concerns a class of isotropic two-dimensional flows for which the diffusivity can be computed explicitly. Such flows consist of arrays of non-overlapping, similar vortices separated by an intricate array of vortex sheets. These models have the property that the net diffusion-advection resulting from all vortices but one has exactly the same effect on the remaining vortex as that of a homogeneous diffusive medium with enhanced diffusivity $\kappa^{*}$, satisfying

$$
\kappa * \propto \kappa_{0} P e^{1 / 2}
$$

as $P e \rightarrow \infty$ or $\kappa_{0} \rightarrow 0$. These examples provide evidence of the fact that intricate vortex sheets, or narrow boundary layers with high Eulerian velocity connecting stagnant regions, can produce a strong enhancement of the macroscopic diffusion rate. The importance of boundary layers in the long-time behavior of incompressible flow was recognized by Childress and Soward [18] for two dimensional Beltrami flows and by Young et al [2] for dispersion in convection rolls; see also Shraiman [43] and Rosenbluth 'et al. [44].

VI.1. Definition of the Stream Functions. To construct the stream functions we consider the following geometric picture. Let $\left\{D_{\alpha}\right\}, \alpha=1,2,3, \ldots$ be a spatially homogeneous, isotropic tiling of the two-dimensional plane $\mathbb{R}^{2}$ by non-overlapping disks $D_{\alpha}$. Such an array of disks must necessarily contain elements with arbitrarily small diameter, since any restriction on the smallness of the diameters would result in a packing volume fraction strictly less than unity, but, aside from these basic geometric features, other details of the tiling can be specified arbitrarily. For instance, the tiling can be periodic, quasi-periodic or random. The centers and radii of the disks will be denoted respectively by $\xi_{\alpha}$ and $r_{\alpha}$, $\alpha=1,2,3, \ldots$. (See Fig. 2). 


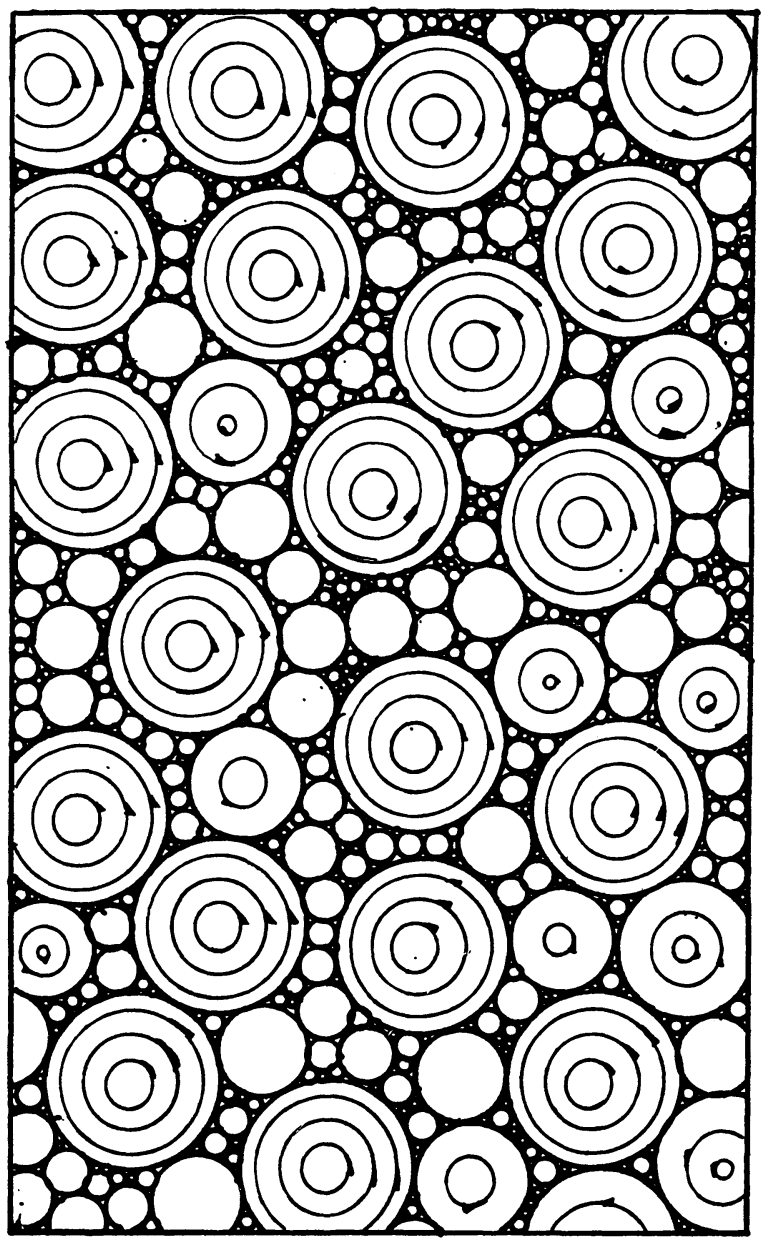

Fig. 2. Schematic representation of the flow geometry corresponding to the examples of Sect. VI. The regions contained between the larger disks become identical, after a scaling transformation, with the original configuration. The streamlines are closed circles drawn in a thin line. The separatrix $\Gamma$, drawn in a thick line, consists of the collection of all circles bounding the disks $D_{v}$

Next, we consider a family $\phi_{r}(\rho), 0 \leqq r \leqq 1$, of functions defined on the interval $0 \leqq \rho \leqq 1$, by

$$
\phi_{r}(\rho)= \begin{cases}\log r & \text { if } \quad 0 \leqq \rho \leqq r \\ \log \rho & \text { if } \quad r \leqq \rho \leqq 1\end{cases}
$$

for $0<r<1$, and, for $r=0$,

$$
\phi_{0}(\rho)=\log \rho, \quad 0<\rho<1 .
$$


We extend the functions $\phi_{r}(\rho)$ by zero for $\rho>1$. For each $r, 0 \leqq r \leqq 1$, we define the stream functions

$$
\psi_{r}(\mathbf{x})=\sum_{\alpha} \phi_{r}\left(\frac{\left|\mathbf{x}-\xi_{\alpha}\right|}{r_{\alpha}}\right), \quad \mathbf{x} \in \mathbb{R}^{2},
$$

and the associated solenoidal velocity fields $\mathbf{u}_{r}(\mathbf{x})$, given by

$$
\begin{aligned}
\mathbf{u}_{r}(\mathbf{x}) & =\sum_{\alpha}\left(\begin{array}{c}
\frac{\partial}{\partial x_{2}} \\
-\frac{\partial}{\partial x_{1}}
\end{array}\right) \phi_{r}\left(\frac{\left|\mathbf{x}-\xi_{\alpha}\right|}{r_{\alpha}}\right) \\
& =\sum_{\alpha} \frac{1}{r_{\alpha}} \phi_{r}^{\prime}\left(\frac{\left|\mathbf{x}-\xi_{\alpha}\right|}{r_{\alpha}}\right)\left[\begin{array}{c}
\frac{x_{2}-\xi_{2 \alpha}}{\left|\mathbf{x}-\xi_{\alpha}\right|} \\
\frac{x_{1}-\underline{\xi}_{1 \alpha}}{\left|\mathbf{x}-\xi_{\alpha}\right|}
\end{array}\right] .
\end{aligned}
$$

The finiteness of $\phi_{r}(\rho)$ for $\rho \approx 1$ ensures that $\psi_{r}(\mathbf{x})$ is continuous across the curve $\Lambda$ consisting of the set-theoretic union of the circles $\left\{x:\left|\mathbf{x}-\xi_{\alpha}\right|=r_{\alpha}\right\}$, as $\alpha$ varies. However, the gradient of $\psi_{r}(\mathbf{x})$, and hence $\mathbf{u}_{r}(\mathbf{x})$ will be discontinuous along $\Lambda$. This set is thus a spatially homogeneous vortex sheet, i.e. a region along which the vorticity

$$
\omega_{r}(x)=\Delta \psi_{r}(\mathbf{x})=\nabla \times \mathbf{u}_{r}(\mathbf{x})
$$

concentrates. In fact, the vorticity of $\mathbf{u}_{r}$ is a measure concentrated on $\Lambda$, as well as on the union of the "internal" circles $\left\{\mathbf{x}:\left|\mathbf{x}-\xi_{\alpha}\right|=r r_{\alpha}\right\}$, for $r>0$, and on the points $\xi_{\alpha}$ for $r=0$. The importance of $\Lambda$ stems from the fact that large-scale motion must necessarily take place by crossing $\Lambda$ and that, since all streamlines $\psi_{r}=$ const. are closed circles, for small values of $\kappa_{0}$, the flow takes place essentially along a thin layer around $\Lambda$.

In the present context it is appropriate to interpret the averaging of spatially homogeneous quantities (such as $\psi_{r}(\mathbf{x}), \psi_{r}^{2}(\mathbf{x}), \mathbf{u}_{r}(\mathbf{x})$, etc.) as Bohr averaging, i.e.,

$$
\langle f\rangle=\lim _{R \rightarrow \infty} \frac{1}{4 \mathrm{R}^{2}} \int_{-R}^{R} \int_{-R}^{R} f\left(x_{1}, x_{2}\right) d x_{1} d x_{2} .
$$

It is then easy to check that

$$
\begin{gathered}
\left\langle\psi_{r}\right\rangle=2 \int_{0}^{1} \phi_{r}(\rho) \rho d \rho=-\frac{1}{2}\left(1-r^{2}\right) . \\
\left\langle\psi_{r}^{2}\right\rangle=2 \int_{0}^{1} \phi_{r}^{2}(\rho) \rho d \rho=r^{2} \ln r+\frac{1}{2}\left(1-r^{2}\right),
\end{gathered}
$$

and

$$
\left\langle\left|\mathbf{u}_{r}\right|^{2}\right\rangle=2 \int_{\theta}^{1}\left(\phi_{r}^{\prime}(\rho)\right)^{2} \rho d \rho=2 \ln \frac{1}{r} .
$$


In particular, the Péclet number associated with the advection diffusion equation

$$
\frac{\partial T}{\partial t}+\mathbf{u}_{r} \cdot \nabla T=\kappa_{0} \Delta T
$$

is given by

$$
\begin{aligned}
P e & =\frac{1}{\kappa_{0}}\left[\left\langle\psi_{r}^{2}\right\rangle-\left\langle\psi_{r}\right\rangle^{2}\right]^{1 / 2} \\
& =\frac{1}{\kappa_{0}}\left[r^{2} \ln r+\frac{1}{2}\left(1-r^{2}\right)-\frac{1}{4}\left(1-r^{2}\right)^{2}\right]^{1 / 2} .
\end{aligned}
$$

We note that, since the velocity field is discontinuous on a set of dimension one, the Lagrangian description of the flow in terms of the stochastic differential equation

$$
d \mathbf{x}(t)=\sqrt{2 \kappa_{0}} d \underset{\sim}{\beta}(t)+\mathbf{u}_{r}(\mathbf{x}(t)) d t
$$

is no longer valid. However the Eulerian description in terms of (6.11) is welldefined. In fact, for $r>0, \psi_{r}(\mathbf{x})$ is uniformly bounded. Since (6.11) can be rewritten in the equivalent form

where

$$
\frac{\partial T}{\partial t}=\nabla \cdot\left[\kappa_{0} \mathbf{I}+\psi_{r} \mathbf{J}\right] \nabla T,
$$

$$
\mathbf{J}=\left[\begin{array}{rr}
0 & -1 \\
1 & 0
\end{array}\right],
$$

the Nash-Moser theorem [40] ensures that (6.14) has a (unique) Hölder-continuous transition probability density $p(t, x, y)$ so that $T(x, t)$ satisfies

$$
T(\mathrm{y}, t)=\int p(t-s, x, y) T(x, s) d x .
$$

For $r=0$, the stream function $\psi_{r}(\mathbf{x})$ diverges logarithmically as $\mathbf{x} \rightarrow \xi_{\alpha}$, at all points $\xi_{\alpha}$. Nevertheless, the radial component of the motion within a given disk $D_{\alpha}$ is a Bessel-2 process

$$
d \rho(t)=\sqrt{2 \kappa_{0}} d \beta(t)+\frac{\sqrt{\kappa_{0}}}{\rho(t)} d t,
$$

which never hits the singular point $\rho=0$, with probability one, since it satisfies the same equation as the radial component of a two-dimensional Brownian motion. This guarantees, by the Stroock-Varadhan theory [41], the existence of a unique transition probability function $p(t, x, y)$ such that (6.16) holds for solutions of the advection-diffusion equation (6.11) with $r=0$.

VI.2. Self-Consistent Calculation of $\kappa^{*}$. As a preliminary calculation, we consider the boundary-value problem

$$
\left\{\begin{array}{lll}
\kappa_{0} \Delta \mathbf{T}(\mathbf{x})+\mathbf{u}(\mathbf{x}) \cdot \nabla \mathbf{T}(\mathbf{x})=0 & \text { for } & |\mathbf{x}| \leqq 1 \\
\mathbf{T}(\mathbf{x})=\mathbf{x} & \text { for } & |\mathbf{x}|=1
\end{array}\right.
$$


where

$$
\mathbf{u}(\mathbf{x})=\phi^{\prime}(|\mathbf{x}|)\left(\begin{array}{c}
\frac{x^{2}}{|x|} \\
-\frac{x_{1}}{|x|}
\end{array}\right)
$$

and $\phi(\rho)=\phi_{r}(\rho)$, for some fixed value of the parameter $r, 0 \leqq r<1$. Introducing polar coordinates $\rho, \theta, 0<\theta<2 \pi$, we seek a solution of (6.18) in the form

$$
\mathbf{T}(x)=\left[\begin{array}{l}
T_{1}(\mathbf{x}) \\
T_{2}(\mathbf{x})
\end{array}\right]
$$

with

$$
T_{1}\left(\rho e^{i \theta}\right)+i T_{2}\left(\rho e^{i \theta}\right)=w(\rho) e^{i \theta}
$$

where $w(\rho)$ is an unknown function. Due to (6.19), we have

$$
\mathbf{u}(\mathbf{x}) \cdot \nabla=\frac{\phi^{\prime}(\rho)}{\rho} \frac{\partial}{\partial \theta},
$$

and hence (6.18) can be recast into a two-point boundary-value problem for $w(\rho)$, namely

$$
\begin{aligned}
& w^{\prime \prime}+\frac{1}{\rho} w^{\prime}-\frac{1}{\rho^{2}} w+\frac{i \phi^{\prime}(\rho)}{\rho \kappa_{0}} w=0, \\
& w(0)=0, \quad w(1)=1 .
\end{aligned}
$$

The boundary condition $w(0)=0$ is imposed so that $\nabla \mathbf{T}$ has finite energy; i.e. $\int|\nabla \mathbf{T}|^{2} d \mathbf{x}<\infty$. Multiplying (6.23) by $\bar{w}(\rho)$ (the complex conjugate) and $|x| \leqq 1$

integrating from 0 to 1 with respect to $\rho d \rho$, we obtain, after integration by parts:

$$
\begin{aligned}
w^{\prime}(1)= & \int_{0}^{1} w^{\prime}(\rho) \bar{w}(\rho) \rho d \rho \\
& +\int_{0}^{1}|w(\rho)|^{2} \frac{d \rho}{\rho}-i \int_{0}^{1} \phi^{\prime}(\rho)|w|^{2} d \rho .
\end{aligned}
$$

Hence

$$
\operatorname{Re} w^{\prime}(1)=\int_{0}^{1}\left[\rho\left|w^{\prime}(\rho)\right|^{2}+\rho^{-1}|w(\rho)|^{2}\right] d \rho
$$

and the average energy of $\nabla \mathbf{T}$ on the unit disk $\{|\mathbf{x}| \leqq 1\}$ is related to the function $w$, from (6.21), by the formula

$$
\begin{aligned}
\frac{1}{2 \pi} \int_{|\mathbf{x}| \leqq 1}|\nabla \mathbf{T}(\mathbf{x})|^{2} d \mathbf{x} & =\int_{0}^{1}\left[\rho\left|w^{\prime}(\rho)\right|^{2}+\rho^{-1}|w(\rho)|^{2}\right] d \rho \\
& =\operatorname{Re} w^{\prime}(1) .
\end{aligned}
$$


We now apply an argument due to Hashin and Shtrikman [25], and Schulgasser [26], to show that the effective diffusivity corresponding to (6.11) is given by

$$
\kappa^{*}=\kappa_{0} \operatorname{Re} w^{\prime}(1) .
$$

The argument consists in exhibiting the solution of the basic equation (2.14)(2.18), which is constructed using the field $\nabla \mathbf{T}(\mathbf{x})$. For this, we consider the stationary, matrix-valued field

$$
\mathbf{E}(\mathbf{x})=\sum_{\alpha} \nabla \mathbf{T}\left[r_{\alpha}^{-1}\left(\mathbf{x}-\xi_{\alpha}\right)\right],
$$

where $\nabla \mathbf{T}(x)$ is extended by zero for $|\mathbf{x}|>1$. Since we have

$$
\left.\frac{\partial}{\partial \theta}\left(T_{1}+i T_{2}\right)\right|_{\rho=1}=i w(1) e^{i \theta}=i e^{i \theta},
$$

the tangential component of $\mathbf{E}(\mathbf{x})$ is continuous across $\Lambda$; and hence

$$
\nabla \times \mathbf{E}(\mathbf{x})=0
$$

in the sense of distributions. Moreover, since $\mathbf{T}(\mathbf{x})=\mathbf{x}$ on $|\mathbf{x}|=1$, we have

$$
\frac{1}{\pi} \int_{|\mathbf{x}| \leqq 1} \nabla \mathbf{T}(\mathbf{x}) d \mathbf{x}=\mathbf{I},
$$

and this implies, using (6.28) and Bohr averaging, that

$$
\langle\mathbf{E}\rangle=\mathbf{I} \text {. }
$$

We will verify by an explicit calculation that $w^{\prime}(1)$ is indeed finite, and hence that $\nabla \mathrm{T}$ has finite energy, by (6.26). As a consequence of this and (6.28), we have

$$
\begin{aligned}
\left\langle|\mathbf{E}|^{2}\right\rangle & =\frac{1}{\pi} \int_{|\mathbf{x}| \leqq 1}|\nabla \mathbf{T}(\mathbf{x})|^{2} d \mathbf{x} \\
& =2 \operatorname{Re} w^{\prime}(1),
\end{aligned}
$$

so $\mathbf{E}$ belongs to $L^{2}(\langle\cdot\rangle)$. It remains to verify that $\mathbf{E}$ is a solution of the basic equation $(2.15)$, which we write as

$$
\kappa_{0} \nabla \cdot \mathbf{E}+\mathbf{u}(x) \cdot \mathbf{E}(x)=0 .
$$

To check this, notice that, from (6.18), the equation is satisfied in the interior of each disk. Finally, we compute the jump of the normal component of $\mathbf{E}$ across an arbitrary point of $\Lambda$. We have, for $x=\rho e^{i \theta}, x_{0}=e^{i \theta}$,

$$
\begin{aligned}
\underset{\rho \uparrow 1}{\lim _{\rho} \mathbf{n}(x) \cdot \nabla \mathbf{T}(\mathbf{x})} & =\frac{\partial}{\partial \rho}\left[\begin{array}{l}
T_{1}\left(\rho e^{i \theta}\right) \\
T_{2}\left(\rho e^{i \theta}\right)
\end{array}\right]_{\rho=1} \\
& =\operatorname{Re} w^{\prime}(1)\left(\begin{array}{c}
\cos \theta \\
\sin \theta
\end{array}\right)+\operatorname{Im} w^{\prime}(1)\left(\begin{array}{r}
-\sin \theta \\
\cos \theta
\end{array}\right) \\
& =\operatorname{Re} w^{\prime}(1) \mathbf{n}\left(\mathbf{x}_{0}\right)+\operatorname{Im} w^{\prime}(1) \underset{\sim}{\tau}\left(\mathbf{x}_{0}\right) .
\end{aligned}
$$


Here $\mathbf{n}$ is the outgoing normal vector and $\tau$ is a vector obtained by rotating the normal $\mathbf{n}$ by $90^{\circ}$ counterclockwise. The definition of $\mathbf{E}$ is such that this calculation yields precisely the outgoing normal flux $\mathbf{n} \cdot \mathbf{E}$ at any point on $\Lambda$, from any disk $D_{\alpha}$, and thus we find that

$$
\text { jump of }\left.\mathbf{n} \cdot \mathbf{E}\right|_{\Lambda}=0 .
$$

This shows that $\mathbf{E}$ is the solution of (6.34) and hence of the basic field equation. To compute the effective diffusivity it suffices to observe that

$$
\begin{aligned}
\kappa^{*} & =\frac{1}{2} \operatorname{Tr}\left\langle\left(\kappa_{0} \mathbf{I}+\psi_{r} \mathbf{J}\right) \mathbf{E}\right\rangle \\
& =\frac{1}{2} \operatorname{Tr}\left\langle\mathbf{E}^{T} \cdot\left(\kappa_{0} \mathbf{I}+\psi_{r} \mathbf{J}\right) \mathbf{E}\right\rangle \\
& =\frac{1}{2} \kappa_{0}\left\langle|\mathbf{E}|^{2}\right\rangle=\kappa_{0} \operatorname{Re} w^{\prime}(1),
\end{aligned}
$$

where the Eqs. (6.33), (6.34) and $\nabla \cdot \psi_{r} \mathbf{J}=\mathbf{u}$ were used.

We now solve explicitly the boundary value problem (6.23) for $\phi(\rho)=\phi_{r}(\rho)$, $0 \leqq r<1$. The problem becomes

with

$$
w(\rho)= \begin{cases}w_{1}(\rho), & \text { if } \quad 0<\rho<r \\ w_{2}(\rho), & \text { if } \quad r<\rho<1\end{cases}
$$

$$
\begin{cases}w_{1}^{\prime \prime}(\rho)+\frac{1}{\rho} w_{1}^{\prime}(\rho)-\frac{1}{\rho^{2}} w_{1}(\rho)=0 & 0<\rho<r, \\ w_{1}^{\prime \prime}(\rho)+\frac{1}{\rho} w_{2}^{\prime}(\rho)-\frac{1}{\rho^{2}}\left(1-i \kappa_{0}^{-1}\right) w_{2}(\rho)=0, & r<\rho<1, \\ w_{1}(0)=0, w_{2}(1)=1, & \\ w_{1}(r)=w_{2}(r), w_{1}^{\prime}(r)=w_{2}^{\prime}(r) . & \end{cases}
$$

We find that $w_{1}, w_{2}$ are given by

$$
\begin{aligned}
& w_{1}(\rho)=a \rho, \\
& w_{2}(\rho)=b \rho^{v}+c \rho^{-v}
\end{aligned}
$$

with

$$
v=\sqrt{1-i \kappa^{-1}},
$$

where the real part of $v$ is positive and the coefficients $a, b, c$ are given by

and

$$
\begin{aligned}
& a=\frac{2 v r^{-2}}{(1+v) r^{-v-1}-(1-v) r^{v-1}}, \\
& b=\frac{(1+v) r^{-v-1}}{(1+v) r^{-v-1}-(1-v) r^{v-1}},
\end{aligned}
$$

$$
c=\frac{(1-v) r^{\nu-1}}{(1-v) r^{v-1}-(1+v) r^{-v-1}} .
$$


We obtain

$$
w^{\prime}(1)=v \frac{1+\left(\frac{1-v}{1+v}\right) r^{2 v}}{1-\left(\frac{1-v}{1+v}\right) r^{2 v}}
$$

so, in particular $\left|w^{\prime}(1)\right|$ is finite, as claimed.

Finally, from (6.27) and (6.44), we conclude that the diffusivity corresponding to $\mathbf{u}_{r}(0 \leqq r<1)$ is given by

$$
\kappa^{*}=\kappa_{0} \operatorname{Re}\left[v \frac{1+\left(\frac{1-v}{1+v}\right) r^{2 v}}{1-\left(\frac{1-v}{1+v}\right) r^{2 v}}\right] .
$$

Since $v=\sqrt{1-i \kappa_{0}^{-1}} \cong \frac{1+i}{\kappa_{0} \sqrt{2}}$ for small values of $\kappa_{0}$, we have

$$
\kappa^{*}=\left(\frac{\kappa_{0}}{2}\right)^{1 / 2}(1+o(1))
$$

for all $r<1$. In particular, at $r=0$, we obtain the simple formula

$$
\kappa^{*}=\kappa_{0} \operatorname{Re} v=\sqrt{\frac{k_{0}^{2}+\kappa_{0} \sqrt{1+\kappa_{0}^{2}}}{2}} .
$$

VI.3. Explicit Formula for the Stieltjes Representation Measure for the Case $r=0$. Substituting, in (6.12), $r=0$, we obtain

$$
P e=\frac{1}{2 \kappa_{0}} .
$$

By substituting this value in Eq. (6.47) we obtain the formula

$$
\kappa^{*}=\kappa_{0}\left(\frac{1+\left(1+4 P e^{2}\right)^{1 / 2}}{2}\right)^{1 / 2},
$$

expressing the dependence of $\kappa^{*} / \kappa_{0}$ on the Péclet number. We wish to compute the corresponding representation measure $d v(\tau)$ entering formula (3.3) for the flow with stream function $\psi_{0}(\mathbf{x})$ given by (6.4) with $r=0$. Making the change of variables $\rho=e^{-t}$, and using (6.38) we can rewrite Eq. (6.38) for $r=0$ as

$$
\left\{\begin{array}{l}
\frac{d^{2} w}{d t^{2}}-w+2 i \text { Pe } w=0, \quad 0<t<\infty \\
w(0)=1, \quad w(\infty)=0 .
\end{array}\right.
$$

We set

$$
w=e^{-t}+z(t),
$$


and obtain a homogenous boundary-value problem for the function $z$ :

$$
\left\{\begin{array}{l}
z^{\prime \prime}-z+2 i P e z=-2 i P e e^{-t}, \quad 0<t<\infty \\
z(0)=z(\infty)=0 .
\end{array}\right.
$$

Using the Fourier transform, one finds readily that:

$$
\begin{aligned}
\operatorname{Re} z^{\prime}(0) & =-\frac{1}{\pi} \int_{-\infty}^{+\infty} \frac{4 P e^{2} w^{2} d w}{\left(1+w^{2}\right)\left[\left(1+w^{2}\right)^{2}+4 P e^{2}\right]} \\
& =-\frac{1}{2 \pi} \int_{-2}^{2} \frac{P e^{2}}{1+P e^{2} \tau^{2}}(2-|\tau|)^{1 / 2}|\tau|^{1 / 2} d \tau .
\end{aligned}
$$

Hence, from (6.27) and (6.51), we find

$$
\frac{\kappa^{*}}{\kappa_{0}}=1+\frac{1}{2 \pi} \int_{-2}^{2} \frac{P e^{2}}{1+P e^{2} \tau^{2}}(2-|\tau|)^{1 / 2}|\tau|^{1 / 2} d \tau,
$$

so that the density of the corresponding representation measure is given by

$$
\frac{d v(\tau)}{d \tau}= \begin{cases}\frac{1}{\pi}(2-|t|)^{1 / 2}|t|^{1 / 2}, & \text { if }|t| \leqq 2 \\ 0, & \text { if }|t|>2\end{cases}
$$

\section{Appendix: Proof of the Homogenization Theorem}

We present an essentially self-contained proof of the homogenization theorem for the advection-diffusion equation

assuming that

$$
\begin{aligned}
\frac{\partial T}{\partial t} & =\kappa_{0} \Delta T-\mathbf{u} \cdot \nabla T \\
& =\kappa_{0} \nabla \cdot(\mathbf{I}-P e \mathbf{H}) \cdot \nabla T,
\end{aligned}
$$

$$
\left\langle|\mathbf{u}(x)|^{d / 2+\varepsilon}\right\rangle+\left\langle|\mathbf{H}(x)|^{p}\right\rangle\langle+\infty
$$

for some $\varepsilon>0$, where $p=2+\varepsilon$, if $d=2$ and $p=d$ for $d \geqq 3$ [ $d=$ space dimension].

The proof of follows the almost classical methods in Bensoussan, Lions and Papanicolaou and L. Tartar [12] for averaging equations with rapidly oscillating coefficients. In adapting such a scheme to homogenize equations with random, unbounded coefficients, two difficulties arise. The first difficulty is that the corrector $\chi(x)$ associated with the basic problem described in Sect. 2 can be unboundẽd and is not, in general, a stationary process. In the context of divergenceform, parabolic equations with random, bounded coefficients, this difficulty was overcome by Papanicolaou and Varadhan [10], who formulated the basic problem (2.14)-(2.17) for the field $\mathbf{E}$ and showed that the corresponding corrector has sublinear growth. (See Lemmas 1, 2, 3 in this section.) The second difficulty arising in the context of random advection-diffusion equations is that it may be desirable to consider fields $\mathbf{H}$ and $\mathbf{u}$ that are, in general, unbounded. (Such is the case, for instance, if $\mathbf{u}$ is a Gaussian field.) Nevertheless, under the additional assumption (A.2), a suitable approximation argument applies and we have the 
Homogenization Theorem. Let $D$ be a bounded region of $\mathbb{R}^{d}$ with smooth boundary $\partial D$, and let the functions $T_{\delta}(x, t), \bar{T}(x, t)$ be the solutions of the following initialboundary-value problems:

$$
\begin{cases}\frac{\partial T_{\delta}(x, t)}{\partial \mathrm{t}} & \text { for }(x, t) \text { in } D \times(0,+\infty) \\ =\kappa_{0} \nabla \cdot\left[\left(\mathbf{I}-\operatorname{Pe} \mathbf{H}\left(\frac{x}{\delta}\right)\right) \cdot \nabla T_{\delta}(x, t)\right], & \\ =\kappa_{0} \Delta T_{\delta}-\frac{1}{\delta} \mathbf{u}\left(\frac{x}{\delta}\right) \cdot \nabla T_{\delta} & \text { for } x \in D, t=0 \\ T_{\delta}(x, 0)=T_{0}(x) & \text { for }(x, t) \in \partial D \times(0,+\infty) \\ T_{\delta}(x, t)=0 & \text { for })\end{cases}
$$

and

$$
\begin{cases}\frac{\partial \bar{T}}{\partial t}=\sum_{i j=1}^{d} \kappa_{i j}^{*} \frac{\partial^{2} \bar{T}(x, t)}{\partial x^{i} \partial x^{j}}, & \text { for }(x, t) \in D \times(0,+\infty) \\ T(x, 0)=T_{0}(x), & \text { for } x \in D, \quad t=0, \\ T(x, t)=0, & \text { for }(x, t) \in \partial D \times(0,+\infty),\end{cases}
$$

where $T_{0}(x)$ is a smooth function with compact support defined in $D$. Then, for all functions $\zeta(x, s)$ satisfying

$$
\left\langle\int_{0}^{\infty} \int_{D} \zeta^{2}(x, s) d x d s\right\rangle<+\infty
$$

we have, for all $t>0$,

$$
\left.\lim _{\delta \downarrow 0}\left\langle\int_{0}^{t} \int_{D} / T_{\delta}(x, s)-\bar{T}(x, s)\right) \zeta(x, s) d x d s\right\rangle=0
$$

The function $T_{\delta}(x, t)$ is understood to be a weak solution of (A.3) in the following sense:

$$
T_{\delta} \in C\left([0,+\infty), L^{2}\left(D, L^{2}(\langle\cdot\rangle)\right)\right)
$$

(ii) For all $t>0$,

$$
T_{\delta} \in L^{2}\left([0, t], W_{0}^{1}\left(D, L^{2}(\langle\cdot\rangle)\right)\right)
$$

(iii) For all $\zeta(x, s) \in C_{0}^{\infty}\left(D \times[0, T], L^{\infty}(\langle\cdot\rangle)\right)$, we have 


$$
\begin{aligned}
& \left\langle\int_{0}^{t} \int_{D} T_{\delta}(x, s) \partial_{s} \zeta(x, s) d x d s\right\rangle \\
& =\kappa_{0}\left\langle\int_{0}^{t} \int_{D}^{t} \nabla T_{\delta}(x, s) \cdot \nabla \zeta(x, s) d x d s\right\rangle \\
& \quad+\left\langle\int_{0}^{t} \int_{D}^{t} \nabla T_{\delta}(x, s) \cdot \frac{1}{\delta} \mathbf{u}\left(\frac{x}{\delta}\right) \zeta(x, s) d x d s\right\rangle .
\end{aligned}
$$

(iv) The initial condition is understood in the $L^{2}$-sense, i.e.

$$
\lim _{t \downarrow 0}\left\langle\int_{D}\left|T_{\delta}(x, t)-T_{0}(x)\right|^{2} d x\right\rangle=0
$$

For a definition and discussion of the spaces $L^{2}\left(D, L^{2}(\langle\cdot\rangle)\right)$ and $W_{0}^{1}\left(D, L^{2}(\langle\cdot\rangle)\right)$ the reader is referred to Papanicolaou and Varadhan [10].

It can be shown that problem (A.3) admits a unique weak solution satisfying (A.7)-(A.10). This is an immediate consequence of the classical theory of parabolic equations with nonsmooth coefficients [42], since from (A.2) the first-order term $\mathbf{u}(x / \delta)$ satisfies the integrability condition

$$
x \mapsto \mathbf{u}\left(\frac{x}{\delta}\right) \in L^{d / 2+\varepsilon}(D), \quad \text { with probability one . }
$$

Moreover, it can be shown that, under the condition (A.11), the solution $T_{\delta}(x, t)$ satisfies

$$
\sup _{\substack{x \in D \\ t>0}}\left|T_{\delta}(x, t)\right| \leqq\left\|T_{0}\right\|_{L^{\infty}(D)},
$$

as well as the energy identity

$$
\int_{D}\left|T_{\delta}(x, t)\right|^{2} d x+\kappa_{0} \int_{0}^{t} \int_{D}\left|\nabla T_{\delta}(x, s)\right|^{2} d x d s=\int_{D}\left|T_{0}(x)\right|^{2} d x
$$

with probability one (see [42], Chap. III, Theorem 7.2). Averaging (A.13) over the velocity statistics, we obtain the integrated energy identity

$$
\begin{aligned}
\left\langle\int_{D}\left|T_{\delta}(x, t)\right|^{2} d x\right\rangle & +\kappa_{0}\left\langle\int_{0}^{t} \int\left|\nabla T_{\delta}(x, s)\right|^{2} d x d s\right\rangle \\
& =\left\langle\int_{D}\left|T_{0}(x)\right|^{2} d x\right\rangle .
\end{aligned}
$$

In addition to these properties of the solution $T_{\delta}(x, t)$ of (A.3), we will need certain asymptotic results for rapidly oscillating random functions in $L^{2}\left(D, L^{2}(\langle\cdot\rangle)\right.$ ), and for the correctors $\chi$ appearing in the formal expansion (2.13). Such results are due to Varadhan and Papanicolaou [10], and are presented here for completeness' sake. Our exposition follows their work closely. 
The reader interested in a probabilistic version of the homogenization theorem can consult the very interesting article of K. Oelschläger [13] that considers random, unbounded $\mathbf{H}$-fields, under somewhat different assumptions than the ones of this paper.

Step. 1. Auxiliary Results and Properties of the Correctors

Lemma 1. Let $f(x) \in L^{2}(\langle\cdot\rangle)$ be a (scalar) random stationary process. Define, for $\alpha=1,2, \ldots, d$, the functions

$$
g_{\alpha}(x)=\int_{\mathbb{R}^{d-\{0\}}} \frac{e^{i k \cdot x}-1}{|k|^{2}} i k_{\alpha} d \hat{f}(k),
$$

where $d \hat{f}(k)$ is the Fourier transform, or spectral measure, of the random process $f(x)$. Then, for all $M>0$,

$$
\lim _{\delta \downarrow 0} \sup _{|x| \leqq M}\left\langle\delta^{2}\left|g_{\alpha}\left(\frac{x}{\delta}\right)\right|^{2}\right\rangle=0 .
$$

Proof. From (A.14), we have

and hence

$$
\begin{aligned}
\left\langle\left|g_{\alpha}(x)\right|^{2}\right\rangle & =\int_{\mathbb{R}^{d-\{0\}}} \frac{\left|e^{i k \cdot x}-1\right|^{2}}{|k|^{2}} \frac{\left\langle\left|k_{\alpha} d \hat{f}(k)\right|^{2}\right\rangle}{|k|^{2}} \\
& \leqq \int_{\mathbb{R}^{d}-\{0\}} \frac{\left|e^{i k \cdot x}-1\right|^{2}}{|k|^{2}}\left\langle|d \hat{f}(k)|^{2}\right\rangle,
\end{aligned}
$$

$$
\begin{aligned}
& \sup _{|x| \leqq M}\left\langle\delta^{2}\left|g_{\alpha}\left(\frac{x}{\delta}\right)\right|^{2}\right\rangle \\
& \leqq \sup _{|x| \leqq M} \int_{\mathbb{R}^{d-\{0\}}} \frac{\mid e^{i(k \cdot x) \delta^{-1}-\left.1\right|^{2}}}{\left|k \delta^{-1}\right|^{2}}\left\langle|d \hat{f}(k)|^{2}\right\rangle \\
& \quad \leqq \int_{\mathbb{R}^{d}-\{0\}}\left[\max _{|x| \leqq M} \frac{\sin ^{2}(k \cdot x / 2 \delta)}{\left|k \delta^{-1}\right|^{2}}\right]\left\langle|d \hat{f}(k)|^{2}\right\rangle .
\end{aligned}
$$

The integrand in (A.17) satisfies

$$
f_{M, \delta}(k) \equiv \max _{|x| \leqq M} \frac{\sin ^{2}(k \cdot x / 2 \delta)}{\left|k \delta^{-1}\right|^{2}} \leqq \frac{M^{2}}{4}
$$

for all $k \neq 0$, as well as

$$
\lim _{\delta \downarrow 0} f_{M, \delta}(k)=0, \quad \text { pointwise . }
$$

Therefore, we can apply the Lebesgue dominated convergence theorem to conclude that, from (A.17), we have $\lim _{\delta \downarrow 0} \sup _{|x| \leqq M}\left(\delta^{2}\left|g_{\alpha}\left(\frac{x}{\delta}\right)\right|^{2}\right)=0$. 
The following lemma gives a useful weak convergence result for stationary, rapidly oscillating functions, viewed as elements of $L^{2}\left(D, L^{2}(\langle\cdot\rangle)\right)$.

Lemma 2. Let $f$ be an element of $L^{2}(\langle\cdot\rangle)$, satisfying $\langle f\rangle=0$, and suppose that $\left\{\zeta_{\delta}(x)\right\}_{\delta}$ is bounded sequence in $W_{0}^{1}\left(D, L^{2}(\langle\cdot\rangle)\right)$. Then, if

we have

$$
\zeta_{\delta}(x) \rightarrow \bar{\zeta}(x) \text { weakly in } L^{2}\left(D, L^{2}(\langle\cdot\rangle)\right) \text {, }
$$

$$
\lim _{\delta \downarrow 0}\left\langle\int_{D} f\left(\frac{x}{\delta}\right) \zeta_{\delta}(x) d x\right\rangle=\langle f\rangle\left\langle\int_{D} \bar{\zeta}(x) d x\right\rangle
$$

Proof. Since $\mathbf{u}$ is ergodic, we can write

$$
f(x)=\langle f\rangle+\int_{\mathbb{R}^{d}-\{0\}} e^{i k \cdot x} d \hat{f}(k),
$$

where $d \hat{f}(k)$ is the spectral measure of $f$. We define the vector field

$$
\mathbf{G}(x)=\left[\begin{array}{c}
g_{1}(x) \\
\vdots \\
g_{d}(x)
\end{array}\right]
$$

where the functions $g_{\alpha}(x), 0 \leqq \alpha \leqq d$, are defined in (A.14). By construction, the vector field $\mathbf{G}(x)$ satisfies

$$
\nabla \cdot \mathbf{G}(x)=\langle f\rangle-f(x)
$$

Hence,

$$
\begin{aligned}
\left\langle\int_{D} f\left(\frac{x}{\delta}\right) \zeta_{\delta}(x) d x\right\rangle= & \langle f\rangle\left\langle\int_{D} \zeta_{\delta}(x) d x\right\rangle \\
& +\left\langle\int_{D} \delta \mathbf{G}\left(\frac{x}{\delta}\right) \cdot \nabla \zeta_{\delta}(x) d x\right\rangle
\end{aligned}
$$

Clearly, the first term in the right-hand-side of (A.25) converges to

$$
\langle f\rangle\left\langle\int_{D} \bar{\zeta}(x) d x\right\rangle
$$

while the second term can be estimated from above by

$$
(\operatorname{Vol}(D))^{1 / 2} \times \sup _{x \in D}\left\langle\delta^{2}\left|\mathbf{G}\left(\frac{x}{\delta}\right)\right|^{2}\right\rangle^{1 / 2} \times\left(\int_{D}\left|\nabla \zeta_{\delta}(x)\right|^{2} d x\right)^{1 / 2}
$$

a quantity that tends to zero, by Lemma 1 . This concludes the proof. 
The next lemma concerns the corrector $\chi$, appearing in the formal expansion (2.13). Let $\mathbf{E}$ be the solution of the basic problem (2.14)-(2.17), i.e.

$$
\begin{aligned}
\nabla \cdot[(I+P e \mathbf{H}) \cdot \mathbf{E}] & =0, \\
\nabla \times \mathbf{E} & =0, \quad\langle\mathbf{E}\rangle=\mathbf{I} .
\end{aligned}
$$

We define the (vector-valued) corrector $\underset{\sim}{\chi}(\mathbf{x}): \mathbb{R}^{d} \rightarrow \mathbb{R}^{d}$ as the function

$$
\underset{\sim}{\chi}(\mathbf{x})=-\int_{\mathbb{R}^{d-\{0\}}} \frac{\left(e^{i k \cdot x}-1\right)}{|k|^{2}} i k \cdot d \hat{\mathbf{E}}(k) .
$$

Following Papanicolaou and Varadhan [10] we claim that

Lemma 3. The function $\underset{\sim}{\chi}$ satisfies

$$
\nabla \underset{\sim}{\chi}(\mathbf{x})=\mathbf{E}^{\prime}(x)=\mathbf{E}(x)-\mathbf{I},
$$

and, for all $q \leqq 2 d / d(d-2)\left(q \in \mathbb{R}^{+}\right.$if $\left.d=2\right)$ and all $\rho \in C_{0}^{\infty}(D)$, we have

$$
\left\langle\left[\int_{D} \delta^{q}\left|\underset{\sim}{\chi}\left(\frac{x}{\delta}\right)\right|^{q} \rho^{q}(x) d x\right]^{2 / q}\right) \leqq C_{q}<+\infty,
$$

uniformly in the parameter $\delta$.

Proof. Differentiating (A.7) with respect to $x$, we obtain

$$
\begin{aligned}
\nabla \underset{\sim}{\chi}(x) & =\int_{\mathbb{R}^{d-\{0\}}} e^{i k \cdot x}|k|^{-2} k k^{T} d \hat{\mathbf{E}}(k), \\
& =-\Gamma_{\sim}^{\Gamma} \mathbf{E}(x), \quad=\mathbf{E}^{\prime}(x),
\end{aligned}
$$

since $-\Gamma$ (with Fourier symbol $|k|^{-2} k k^{T}$ ) is the projection operator onto the set of curl-free fields with zero mean. This proves claim (i). The second assertion is an immediate consequence of Lemma 1. Finally, to prove (A.32) we introduce the function

$$
\mathbf{f}_{\delta}(x) \equiv \delta \underset{\sim}{\chi}\left(\frac{x}{\delta}\right) \rho(x)
$$

which is an element of $W_{0}^{1}\left(D, L^{2}(\langle\cdot\rangle)\right)$, since

$$
\nabla \mathbf{f}_{\delta}(x)=\nabla \underset{\sim}{\chi}\left(\frac{x}{\delta}\right) \rho(x)+\delta \underset{\sim}{\chi}\left(\frac{x}{\delta}\right) \nabla \rho(x) .
$$


By the Sobolev inequality [42], we have

$$
\left[\int_{\mathbb{R}^{d}} f_{\delta}^{q}(x) d x\right]^{2 / q} \leqq C \int_{\mathbb{R}^{d}}\left|\nabla f_{\delta}(x)\right|^{2} d x,
$$

where $C$ is a numerical constant depending only on $d$. Hence, integrating both sides of this inequality with respect to $\langle\cdot\rangle$, we obtain

$$
\begin{aligned}
& \left\langle\left.\left(\int_{D} \delta^{q} \mid \chi\left(\frac{x}{\delta}\right)^{q} \rho^{q}(x) d x\right)\right|^{2 / q}\right\rangle \\
& \leqq C\left\langle\int_{\mathbb{R}^{d}}\left|\nabla f_{\delta}(x)\right|^{2} d x\right\rangle \\
& \leqq C\left\langle\int_{\mathbb{R}^{d}}\left|\mathbf{E}^{\prime}\left(\frac{x}{\delta}\right)\right|^{2} \rho^{2}(x) d x\right\rangle \\
& \quad+C\left\langle\int_{\mathbb{R}^{d}} \delta^{2}\left|\chi\left(\frac{x}{\delta}\right)\right|^{2}|\nabla \rho(x)|^{2} d x\right\rangle \\
& \leqq C\left\|E^{\prime}\right\|_{L^{2}(\langle\cdot\rangle)}^{2}\|\rho\|_{L^{2}(D)}^{2} \\
& \quad+C\|\nabla \rho\|_{L^{2}(D)}^{2} \sup _{|x| \in D}\left\langle\delta^{2}\left|\chi\left(\frac{x}{\delta}\right)\right|^{2}\right\rangle,
\end{aligned}
$$

which is bounded independently of $\delta$. This concludes the proof of Lemma 3 .

Step 2. The Energy Argument. From the integrated energy identity (A.13') we conclude that there exists a subsequence $T_{\delta_{n}}(x, t), n \geqq 1-$ which we still denote by $T_{\delta}(x, t)$ for simplicity - and a function $\bar{T}(x, t)$ such that

$$
T_{\delta} \rightarrow \bar{T} \text { weakly in } L^{2}\left([0, T], W_{0}^{1}\left(D, L^{2}(\langle\cdot\rangle)\right)\right) \text {, }
$$

for all $T>0$.

Let us consider the vector field

$$
\mathbf{q}_{\delta}(x, s)=\left[\mathbf{I}-\operatorname{Pe} \mathbf{H}\left(\frac{x}{\delta}\right)\right] \cdot \nabla T_{\delta}(x, s) .
$$

The next lemma establishes a bound on $\mathbf{q}_{\delta}(x, s)$ in a suitable norm, which is independent of $\delta$.

Lemma 4. There exists a positive constant, $C$, depending only on $t,\left\|T_{0}\right\|_{L^{2}(D)}$, Vol $D$, and $\|\mathbf{H}\|_{L^{p(\langle\cdot\rangle)}}($ with $p=d$ if $d \geqq 3$ and $p=2+\varepsilon$ if $d=2)$, and not depending on $\delta$, such that

$$
\left\langle\left(\int_{0}^{t} \int_{D}\left|\mathbf{q}_{\delta}(x, s)\right|^{r} d x d s\right)^{2 / r}\right\rangle \leqq C,
$$

with $r=2 d /(d+2)$ if $d \geqq 3$ and $r=(4+2 \varepsilon) /(4+\varepsilon)$ if $d=2$. 
Proof. By Hölder's inequality, for all $r \geqq 1$,

$$
\begin{aligned}
& \left(\int_{0}^{t} \int_{D}\left|\mathbf{q}_{\delta}(x, s)\right|^{r} d x d s\right)^{2 / r} \\
& \leqq\left(\int_{0}^{t} \int_{D}\left|I-P e \mathbf{H}\left(\frac{x}{\delta}\right)\right|^{p} d x d s\right)^{2 / p}\left(\int_{0}^{t} \int_{D}\left|\nabla T_{\delta}(x, s)\right|^{2} d x d s\right),
\end{aligned}
$$

for $p$ such that $1 / p+1 / 2=1 / r$. We observe that, by the (pointwise) energy identity (A.13), $\int_{0}^{t} \int_{D}\left|\nabla T_{\delta}(x, s)\right|^{2} d x d s$ is uniformly bounded, with probability one, in terms of the $L^{2}$-norm of the initial data. Accordingly, taking $p$ and $r$ as in the statement of this lemma, we have

$$
\begin{aligned}
& \left\langle\left(\int_{0}^{t} \int_{D}\left|\mathbf{q}_{\delta}(x, s)\right|^{r} d x d s\right)^{2 / r}\right\rangle \\
& \leqq t^{2 / p} \frac{\left\|T_{0}\right\|_{L^{2}(D)}^{2}}{\kappa_{0}} \times\left\langle\left(\int_{D}\left|\mathbf{I}-\operatorname{Pe} \mathbf{H}\left(\frac{x}{\delta}\right)\right|^{p} d x\right)^{2 / p}\right\rangle \\
& \leqq t^{2 / p} \frac{\left\|\mathbf{T}_{0}\right\|_{L^{2}(D)}^{2}}{\kappa_{0}} \times\left\langle\int_{D}\left|\mathbf{I}-\operatorname{Pe} \mathbf{H}\left(\frac{x}{\delta}\right)\right|^{p} d x\right\rangle^{2 / p} \\
& \left.=t^{2 / p} \frac{\left\|T_{0}\right\|_{L^{2}(D)}^{2}}{\kappa_{0}} \times(\operatorname{Vol} D)\right)^{2 / p}\left\langle|I-\operatorname{Pe} \mathbf{H}(\cdot)|^{p}\right\rangle^{2 / p} .
\end{aligned}
$$

In deriving this estimate, we used Jensen's inequality and the fact that $\mathbf{H}$ is a stationary random process. This concludes the proof of Lemma 4.

As a consequence of Lemma $4,\left\{\mathbf{q}_{\delta}(x, s)\right\}_{s>0}$ is a bounded set in the reflexive Banach space $L^{2}\left(\langle\cdot\rangle, L^{p}([0, t] \times D)\right)$ and hence has a weakly convergent subsequence, $\left\{\mathbf{q}_{\delta_{n}}(x, s)\right\} ; \boldsymbol{\delta}_{n} \rightarrow 0$. Let us denote the corresponding limit by $\overline{\mathbf{q}}(x, s)$. Taking the limit in Eq. (A.9) as $\delta_{n} \rightarrow 0$, and using the fact that $\mathbf{u}(x)=\kappa_{0} P e \nabla \cdot \mathbf{H}$, we obtain

$$
\left\langle\int_{0}^{t} \int_{D} \bar{T}(x, s) \partial_{s} \zeta(x, s) d x d s\right\rangle=\kappa_{0}\left\langle\int_{0}^{t} \int_{D}(\nabla \zeta(x, s))^{T} \cdot \overline{\mathbf{q}}(x, s) d x d s\right\rangle
$$

for any function $\zeta(x, s) \in C_{0}^{\infty}\left(D \times[0, T] ; L^{\infty}(\langle\cdot\rangle)\right)$. This can be interpreted as statement that $\bar{T}(x, s), \overline{\mathbf{q}}(x, s)$ satisfy, formally, the equation

$$
\frac{\partial}{\partial s} \bar{T}(x, s)=\kappa_{0} \nabla \cdot \overline{\mathbf{q}}(x, s) .
$$

The problem of interest is to identify $\kappa_{0} \overline{\mathbf{q}}(x, s)$ as the "homogenized flux" corresponding to (A.4), i.e. to show that

$$
\kappa_{0} \overline{\mathbf{q}}(x, s)={\underset{\sim}{\kappa}}^{*} \cdot \nabla \bar{T}(x, s)
$$


with ${\underset{\sim}{\kappa}}^{*}={\underset{\sim}{\kappa}}_{0}\langle(\mathbf{I}+P e \mathbf{H}) \mathbf{E}\rangle$. For this, we follow the classical approach due to Tartar [11], which consists in introducing the test functions

$$
\zeta_{\delta}(x, s)=\left[\mathbf{x} \cdot \mathbf{v}+\delta \underset{\sim}{\underset{\sim}{\chi}}\left(\frac{x}{\delta}\right)\right] \zeta(x, s)
$$

where $\mathbf{v} \in \mathbb{R}^{d}$ and $\zeta(x, s) \in C_{0}^{\infty}\left([0, t] \times D ; L^{\infty}(\langle\cdot\rangle)\right)$.

Using the incompressibility of $\mathbf{u}$, we can rewrite the identity (A.9) as

$$
\begin{aligned}
& \left\langle\int_{0}^{t} \int_{D} T_{\delta}(x, s) \partial_{s} \zeta(x, s) d x d s\right\rangle \\
& =\kappa_{0}\left\langle\int_{0}^{t} \int_{D}\left(\nabla T_{\delta}(x, s)\right)^{T} \cdot(\nabla \zeta(x, s)) d x d s\right\rangle \\
& \quad-\left\langle\int_{0}^{t} \int_{D} T_{\delta}(x, s) \frac{1}{\delta} \mathbf{u}\left(\frac{x}{\delta}\right) \cdot \nabla \zeta(x, s) d x d s\right\rangle .
\end{aligned}
$$

Substituting the test function $\zeta_{\delta}(x, s)$ in this identity, we obtain

$$
\begin{aligned}
\left\langle\int_{0}^{t} \int_{D} T_{\delta}(x, s)\left[\mathbf{x} \cdot \mathbf{v}+\underset{\sim}{\underset{\sim}{\chi}}\left(\frac{x}{\delta}\right) \cdot \mathbf{v}\right] \partial_{s} \zeta(x, s) d x d s\right\rangle \\
=\kappa_{0}\left\langle\int_{0}^{t} \int_{D}\left(\nabla T_{\delta}(x, s)^{T} \cdot \nabla \zeta(x, s)\left[\mathbf{x} \cdot \mathbf{v}+\underset{\sim}{\underset{\sim}{\chi}}\left(\frac{x}{\delta}\right) \cdot \mathbf{v}\right] d x d s\right\rangle\right. \\
+\kappa_{0}\left\langle\int_{0}^{t} \int_{D}\left(\nabla T_{\delta}(x, s)\right)^{T} \cdot \mathbf{E}\left(\frac{x}{\delta}\right) \cdot \mathbf{v} \zeta(x, s) d x d s\right\rangle \\
-\left\langle\int_{0}^{t} \int_{D} T_{\delta}(x, s) \zeta(x, s) \frac{1}{\delta} \mathbf{u}\left(\frac{x}{\delta}\right) \cdot \mathbf{E}\left(\frac{x}{\delta}\right) \cdot \mathbf{v} d x d s\right\rangle \\
\quad-\left\langle\int_{0}^{t} \int_{D} T_{\delta}(x, s)\left[\mathbf{x} \cdot \mathbf{v}+\underset{\sim}{\underset{\sim}{\chi}}\left(\frac{x}{\delta}\right) \cdot \mathbf{v}\right] \frac{1}{\delta} \mathbf{u}\left(\frac{x}{\delta}\right) \cdot \nabla \zeta(x, s)\right\rangle
\end{aligned}
$$

where we used the identity (cf. Lemma 3),

$$
\nabla\left[\mathbf{x} \cdot \mathbf{v}+\delta \underset{\sim}{\chi}\left(\frac{x}{\delta}\right) \cdot \mathbf{v}\right]=\mathbf{E}\left(\frac{x}{\delta}\right) \cdot \mathbf{v}
$$

Note that, by (A.12), $T_{\delta}$ is uniformly bounded, so we can in fact introduce the unbounded test function $\zeta_{\delta}$ in (A.48) and obtain (A.49). Using integration by parts in (A.49), we have 


$$
\begin{aligned}
& \left\langle\int_{0}^{t} \int_{D} T_{\delta}(x, s)\left[\mathbf{x} \cdot \mathbf{v}+\delta \underset{\sim}{\underset{\gamma}{\chi}}\left(\frac{x}{\delta}\right) \cdot \mathbf{v}\right] \frac{1}{\delta} u\left(\frac{x}{\delta}\right) \cdot \nabla \zeta(x, s) d x d s\right\rangle=\kappa_{0} P e \\
& \times\left\langle\int_{0}^{t} \int_{D} T_{\delta}(x, s)\left[\mathbf{x} \cdot \mathbf{v}+\delta \underset{\sim}{\underset{\delta}{\chi}}\left(\frac{x}{\delta}\right) \cdot \mathbf{v}\right] \nabla \cdot\left[\mathbf{H}\left(\frac{x}{\delta}\right) \cdot \nabla \zeta(x, s) d x d s\right\rangle\right. \\
& =\kappa_{0} P e\left\langle\int_{0}^{t} \int_{D}\left[\mathbf{x} \cdot \mathbf{v}+\delta \underset{\sim}{\chi}\left(\frac{x}{\delta}\right) \cdot \mathbf{v}\right](\nabla \zeta(x, s))^{T}\right. \\
& \left.\cdot \mathbf{H}\left(\frac{x}{\delta}\right) \cdot \nabla T_{\delta}(x, s) d x d s\right\rangle \\
& +\kappa_{0} P e\left\langle\int_{0}^{t} \int_{D}(\nabla \zeta(x, s))^{T} \cdot \mathbf{H}\left(\frac{x}{\delta}\right) \cdot \mathbf{E}\left(\frac{x}{\delta}\right) \cdot \mathbf{v} T_{\delta}(x, s) d x d s\right\rangle .
\end{aligned}
$$

Moreover,

$$
\begin{aligned}
\kappa_{0} & \left\langle\int_{0}^{t} \int_{D}\left(\nabla T_{\delta}(x, s)\right)^{T} \cdot \mathbf{E}\left(\frac{x}{\delta}\right) \cdot \mathbf{v} \zeta(x, s) d x d s\right\rangle \\
= & \kappa_{0}\left\langle\int_{0}^{t} \int_{D}\left[\nabla\left(T_{\delta}(x, s) \zeta(x, s)\right)\right]^{T} \cdot \mathbf{E}\left(\frac{x}{\delta}\right) \cdot \mathbf{v} d x d s\right\rangle \\
& -\kappa_{0}\left\langle\int_{0}^{t} \int_{D}(\nabla \zeta(x, s))^{T} \cdot \mathbf{E}\left(\frac{x}{\delta}\right) \cdot \mathbf{v} T_{\delta}(x, s) d x d s\right\rangle .
\end{aligned}
$$

Substituting expressions (A.51) and (A.52) in (A.49) we conclude that

$$
\begin{aligned}
& \left\langle\int_{0}^{t} \int_{D} T_{\delta}(x, s)\left[\mathbf{x} \cdot \mathbf{v}+\underset{\sim}{\underset{\chi}{\chi}}\left(\frac{x}{\delta}\right) \cdot \mathbf{v}\right] \partial_{s} \zeta(x, s) d x d s\right\rangle \\
& =\kappa_{0}\left\langle\int_{0}^{t} \int_{D}\left[\mathbf{x} \cdot \mathbf{v}+\delta \underset{\sim}{\underset{\chi}{\chi}}\left(\frac{x}{\delta}\right) \cdot \mathbf{v}\right](\nabla \zeta(x, s))^{T}\right. \\
& \left.\cdot\left(\mathbf{I}-P e \mathbf{H}\left(\frac{x}{\delta}\right)\right) \cdot \nabla T_{\delta}(x, s) d x d s\right\rangle \\
& \quad-\kappa_{0}\left\langle\int_{0}^{t} \int_{D}(\nabla \zeta(x, s))^{T}\left(\mathbf{I}+P e \mathbf{H}\left(\frac{x}{\delta}\right)\right) \mathbf{E}\left(\frac{x}{\delta}\right) \cdot \mathbf{v} T_{\delta}(x, s) d x d s\right\rangle \\
& +\kappa_{0}\left\langle\int_{0}^{t} \int_{D}\left[\nabla\left(T_{\delta}(x, s) \zeta(x, s)\right)\right]^{T} \cdot \mathbf{E}\left(\frac{x}{\delta}\right) \cdot \mathbf{v} d x d s\right\rangle \\
& \quad-\left\langle\int_{0}^{t} \int_{D} T_{\delta}(x, s) \zeta(x, s) \frac{1}{\delta} \mathbf{u}_{\delta}\left(\frac{x}{\delta}\right) \cdot \mathbf{E}\left(\frac{x}{\delta}\right) \cdot \mathbf{v} d x d s\right\rangle .
\end{aligned}
$$


The sum of the last two terms on the right-hand side of (A.53) can be computed using the equation satisfied by $\mathbf{E}$. For this, let $\left\{\psi_{n}(x, s)\right\}$ denote a sequence of smooth functions satisfying

$$
\left|\psi_{n}(x, s)\right| \leqq C_{1}, \quad x \in D, \quad 0<s<t,
$$

with probability one, for some constant $C_{1}$, and

$$
\psi_{n} \rightarrow T_{\delta} \zeta \text { strongly in } L^{2}\left([0, T], W_{0}^{1}\left(D, L^{2}(\langle\cdot\rangle)\right)\right) .
$$

Then, we have

$$
\begin{aligned}
\kappa_{0}\left\langle\int_{0}^{t} \int_{D}\left[\nabla\left(T_{\delta}(x, s) \zeta(x, s)\right)\right]^{T} \cdot \mathbf{E}\left(\frac{x}{\delta}\right) \cdot \mathbf{v} d x d s\right\rangle \\
-\left\langle\int_{0}^{t} \int_{D} T_{\delta}(x, s) \zeta(x, s) \frac{1}{\delta} \mathbf{u}_{\delta}\left(\frac{x}{\delta}\right) \cdot \mathbf{E}\left(\frac{x}{\delta}\right) \cdot \mathbf{v} d x d s\right\rangle \\
=\lim _{n \rightarrow \infty}\left[\kappa_{0}\left\langle\int_{0}^{t} \int_{D}\left(\nabla \psi_{n}(x, s)\right)^{T} \cdot \mathbf{E}\left(\frac{x}{\delta}\right) \cdot \mathbf{v} d x d s\right\rangle\right. \\
\left.\quad-\left\langle\int_{0}^{t} \int_{D} \psi_{n}(x, s) \frac{1}{\delta} \mathbf{u}_{\delta}\left(\frac{x}{\delta}\right) \cdot \mathbf{E}\left(\frac{x}{\delta}\right) \cdot \mathbf{v} d x d s\right\rangle\right] \\
=\lim _{n \rightarrow \infty}\left[\kappa_{0}\left\langle\int_{0}^{t} \int_{D}\left(\nabla \psi_{n}(x, s)\right)^{T} \cdot \mathbf{E}\left(\frac{x}{\delta}\right) \cdot \mathbf{v} d x d s\right\rangle\right. \\
\left.\quad+\kappa_{0} P e\left\langle\int_{0}^{t} \int_{D}\left(\nabla \psi_{n}(x, s)\right)^{T} \cdot \mathbf{H}\left(\frac{x}{\delta}\right) \cdot \mathbf{E}\left(\frac{x}{\delta}\right) \cdot \mathbf{v} d x d s\right\rangle\right] \\
=\lim _{n \rightarrow \infty} \kappa_{0}\left\langle\int_{0}^{t} \int_{D}\left(\nabla \psi_{n}(x, s)\right)^{T} \cdot\left(\mathbf{I}+P e \mathbf{H}\left(\frac{x}{\delta}\right)\right) \cdot \mathbf{E}\left(\frac{x}{\delta}\right) \cdot \mathbf{v} d x d s\right\rangle \\
=0,
\end{aligned}
$$

since $\nabla \cdot[(\mathbf{I}+P e \mathbf{H}) \mathbf{E}]=0$ in the sense of distributions.

Summarizing, we have shown that, from (A.53), the solution $T_{\delta}(x, s)$ of the problem (A.3) and the vector field $\mathbf{q}_{\delta}(x, s)$ satisfy, for all smooth test functions $\zeta(x, s)$, the equation

$$
\begin{aligned}
&\left\langle\int_{0}^{t} \int_{D} T_{\delta}(x, s)\right. {\left.\left[\mathbf{x} \cdot \mathbf{v}+\delta \underset{\sim}{\chi}\left(\frac{x}{\delta}\right) \cdot \mathbf{v}\right] \partial_{s} \zeta(x, s) d x d s\right\rangle } \\
&= \kappa_{0}\left\langle\int_{0}^{t} \int_{D}\left[\mathbf{x} \cdot \mathbf{v}+\delta{\underset{\sim}{\chi}}^{\chi}\left(\frac{x}{\delta}\right) \cdot \mathbf{v}\right](\nabla \zeta(x, s))^{T} \cdot \mathbf{q}_{\delta}(x, s) d x d s\right\rangle \\
&- \kappa_{0}\left\langle\int_{0}^{t} \int_{D}(\nabla \zeta(x, s))^{T} \cdot\left(\mathbf{I}+P e \mathbf{H}\left(\frac{x}{\delta}\right)\right)\right. \\
&\left.\cdot \mathbf{E}\left(\frac{x}{\delta}\right) \cdot \mathbf{v} T_{\delta}(x, s) d x d s\right\rangle .
\end{aligned}
$$


We wish to pass to the limit in this identity as $\delta \rightarrow 0$ (along a subsequence). Clearly,

$$
\begin{aligned}
\lim _{\delta \downarrow 0} & \left\langle\int_{0}^{t} \int_{D} T_{\delta}(x, s)\left[\mathbf{x} \cdot \mathbf{v}+\underset{\sim}{\boldsymbol{\chi}} \underset{\sim}{(}\left(\frac{x}{\delta}\right) \cdot \mathbf{v}\right] \partial_{s} \zeta(x, s) d x d s\right\rangle \\
& =\left\langle\int_{0}^{t} \int_{D} \bar{T}(x, s)(\mathbf{x} \cdot \mathbf{v}) \partial_{s} \zeta(x, s) d x d s\right\rangle .
\end{aligned}
$$

To pass to the limit in the first summand of the right-hand side of (A.57), we write

$$
\begin{aligned}
\mathbf{q}_{\delta} & (x, s) \\
& =\left(\mathbf{I}-P e \mathbf{H}^{(n)}\left(\frac{x}{\delta}\right)\right) \cdot \nabla T_{\delta}(x, s)+P e\left[\mathbf{H}\left(\frac{x}{\delta}\right)-\mathbf{H}^{(n)}\left(\frac{x}{\delta}\right)\right] \cdot \nabla T_{\delta}(x, s) \\
& =\mathbf{q}_{\delta}^{(n)}(x, s)+P e\left(\mathbf{H}\left(\frac{x}{\delta}\right)-\mathbf{H}^{(n)}\left(\frac{x}{\delta}\right)\right) \cdot \nabla T_{\delta}(x, s)
\end{aligned}
$$

where $\mathbf{H}^{(n)}$ is the usual trunction of the $\mathbf{H}$-field. Using the method of Lemma 4, we have the estimate

$$
\left\langle\left(\int_{0}^{t} \int_{D}\left|\mathbf{q}_{\delta}(x, s)-\mathbf{q}_{\delta}^{(n)}(x, s)\right|^{r}\right)^{2 / r}\right\rangle \leqq C_{2}\left\langle\left|\mathbf{H}-\mathbf{H}^{(n)}\right|^{p}\right\rangle^{2 / p},
$$

where $p$ and $r$ are as in Lemma 4. Consequently, if $\overline{\mathbf{q}}^{(n)}(x, s)$ denotes the weak limit of $\mathbf{q}_{\delta}^{(n)}(x, s)$ as $\delta \rightarrow 0$, we have

$$
\lim _{n \rightarrow \infty}\left\langle\left(\int_{0}^{t} \int_{D}\left|\overline{\mathbf{q}}(x, s)-\overline{\mathbf{q}}^{(n)}(x, s)\right|^{r} d x d s\right)^{2 / r}\right\rangle=0 .
$$

Using the decomposition (A.59), we write

$$
\begin{aligned}
& \lim _{\delta \downarrow 0}\left\langle\int_{0}^{t} \int_{D}\left[\mathbf{x} \cdot \mathbf{v}+\delta \underset{\sim}{\chi}\left(\frac{x}{\delta}\right) \cdot \mathbf{v}\right](\nabla \zeta(x, s))^{T} \cdot \mathbf{q}_{\delta}(x, s) d x d s\right\rangle \\
&= \lim _{\delta \downarrow 0}\left\langle\int_{0}^{t} \int_{D}(\mathbf{x} \cdot \mathbf{v})(\nabla \zeta(x, s))^{T} \cdot \mathbf{q}_{\delta}^{(n)}(x, s) d x d s\right\rangle \\
&+\lim _{\delta \downarrow 0}\left\langle\int_{0}^{t} \int_{D}\left(\delta \underset{\sim}{\chi}\left(\frac{x}{\delta}\right) \cdot \mathbf{v}\right)(\nabla \zeta(x, s))^{T} \cdot\left(\mathbf{I}-P e \mathbf{H}^{(n)}\left(\frac{x}{\delta}\right)\right)\right. \\
&\left.\cdot \nabla T_{\delta}(x, s) d x d s\right\rangle \\
& \lim _{\delta \downarrow 0}\langle\left\langle\int_{0}^{t} \int_{D}\left[\mathbf{x} \cdot \mathbf{v}+\delta \underset{\sim}{\chi}\left(\frac{x}{\delta}\right) \cdot \mathbf{v}\right](\nabla \zeta(x, s))^{T}\right. \\
&\left.\cdot\left(\mathbf{q}_{\delta}(x, s)-\mathbf{q}^{(n)}(x, s)\right) d x d s\right\rangle .
\end{aligned}
$$


By Lemma 3, since $\mathbf{H}^{(n)}$ is uniformly bounded, the second summand of the righthand side is equal to zero. Moreover, using (A.60) and (A.61) we can conclude, by letting $n \rightarrow \infty$ in (A.62), that

$$
\begin{aligned}
\lim _{\delta \downarrow 0} & \left\langle\int_{0}^{t} \int_{D}\left[\mathbf{x} \cdot \mathbf{v}+\underset{\sim}{\boldsymbol{\chi}} \underset{\sim}{(}\left(\frac{x}{\delta}\right) \cdot \mathbf{v}\right](\nabla \zeta(x, s))^{T} \cdot \mathbf{q}_{\delta}(x, s) d x d s\right\rangle \\
& =\left\langle\int_{0}^{t} \int_{D}(\mathbf{x} \cdot \mathbf{v})(\nabla \zeta(x, s))^{T} \overline{\mathbf{q}}(x, s) d x d s\right\rangle .
\end{aligned}
$$

Finally, we wish to pass to the limit as $\delta \rightarrow 0$ in the second summand of the righthand side of (A.57). For this, set

$$
\mathbf{f}(\mathbf{x})=(\mathbf{I}+\operatorname{Pe} \mathbf{H}(\mathbf{x}) \cdot \mathbf{E}(x) \cdot \mathbf{v} \text {. }
$$

The function $\mathbf{f}(\mathbf{x})$ is an element of $L^{1}(\langle\cdot\rangle)$. Let $\left\{\mathbf{f}^{(n)}(\mathbf{x})\right\}_{n \geqq 1}$ denote a sequence in $L^{2}(\langle\cdot\rangle)$ such that

$$
\left\langle\left|\mathbf{f}^{(n)}-\mathbf{f}\right|\right\rangle \leqq \frac{1}{n}, \quad n \geqq 1
$$

Accordingly, we have

$$
\begin{aligned}
\lim _{\delta \downarrow 0} & \left.\left\langle\int_{0}^{t} \int_{D}(\nabla \zeta(x, s))\right)^{T} \cdot \mathbf{f}\left(\frac{x}{\delta}\right) T_{\delta}(x, s) d x d s\right\rangle \\
= & \lim _{\delta \downarrow 0}\left\langle\int_{0}^{t} \int_{D}(\nabla \zeta(x, s))^{T} \mathbf{f}^{(n)}\left(\frac{x}{\delta}\right) T_{\delta}(x, s) d x d s\right\rangle \\
& \left.+\lim _{\delta \downarrow 0}\left\langle\int_{0}^{t} \int_{D} \nabla \zeta(x, s)\right)^{T}\left[\mathbf{f}\left(\frac{x}{\delta}\right)-\mathbf{f}^{(n)}\left(\frac{x}{\delta}\right)\right] T_{\delta}(x, s) d x d s\right\rangle .
\end{aligned}
$$

From Lemma 2, we have

$$
\begin{aligned}
\lim _{\delta \downarrow 0} & \left\langle\int_{0}^{t} \int_{D}(\nabla \zeta(x, s))^{T} \cdot \mathbf{f}^{(n)}\left(\frac{x}{\delta}\right) \cdot T_{\delta}(x, s) d x d s\right\rangle \\
& =\left\langle\int_{0}^{t} \int_{D}(\nabla \zeta(x, s))^{T}\left\langle\mathbf{f}^{(n)}\right\rangle \bar{T}(x, s) d x d s\right\rangle .
\end{aligned}
$$

Finally, the remainder satisfies

$$
\begin{aligned}
& \left|\left\langle\int_{0}^{t} \int_{D}(\nabla \zeta(x, s))^{T} \cdot\left(\mathbf{f}\left(\frac{x}{\delta}\right)-\mathbf{f}^{(n)}\left(\frac{x}{\delta}\right)\right) \cdot T_{\delta}(x, s) d x d s\right\rangle\right| \\
& \quad \leqq t(\operatorname{Vol} D)\|\nabla \zeta\|_{\infty}\left\|T_{0}\right\|_{\infty}\left\langle\left|\mathbf{f}-\mathbf{f}^{(n)}\right|\right\rangle \leqq \frac{c}{n},
\end{aligned}
$$

and hence 


$$
\begin{aligned}
\lim _{\delta \downarrow 0} & \left\langle\int_{0}^{t} \int_{D}(\nabla \zeta(x, s))^{T} \cdot \mathbf{f}\left(\frac{x}{\delta}\right) T_{\delta}(x, s) d x d s\right\rangle \\
= & \lim _{n \rightarrow \infty}\left\langle\int_{0}^{t} \int_{D}(\nabla \zeta(x, s))^{T}\left\langle\mathbf{f}^{(n)}\right\rangle \bar{T}(x, s) d x d s\right\rangle \\
= & \frac{1}{\kappa_{0}} \int_{0}^{t} \int_{D}\left\langle(\nabla \zeta(x, s))^{T} \cdot{\underset{\sim}{\mathcal{N}}}^{*} \cdot \mathbf{v} \bar{T}(x, s) d x d s\right\rangle,
\end{aligned}
$$

since $\langle\mathbf{f}\rangle=\langle(\mathbf{I}+P e \mathbf{H}) \cdot \mathbf{E}\rangle \mathbf{v}=\frac{1}{\kappa_{0}} \underset{\sim}{\kappa} * \mathbf{v}$. Putting together (A.58), (A.63) and (A.69) we obtain, from (A.57),

$$
\begin{aligned}
\left\langle\int_{0}^{t} \int_{D} \bar{T}(x, s)(\mathbf{x} \cdot \mathbf{v}) \partial_{s} \zeta(x, s) d x d s\right\rangle \\
=\kappa_{0}\left\langle\left\langle\int_{0}^{t} \int_{D}(\mathbf{x} \cdot \mathbf{v})(\nabla \zeta(x, s))^{T} \cdot \overline{\mathbf{q}}(x, s) d x d s\right\rangle\right. \\
\quad-\left\langle\int_{0}^{t} \int_{D}(\nabla \zeta(x, s))^{T} \cdot{\underset{\sim}{*}}^{*} \cdot \mathbf{v} \bar{T}(x, s) d x d s\right\rangle .
\end{aligned}
$$

But, substituting the test function $\mathbf{x} \cdot \mathbf{v} \zeta(x, s)$ in (A.9) and letting $\delta \rightarrow 0$ yields, as in (A.44),

$$
\begin{aligned}
& \left\langle\int_{0}^{t} \int_{D} \bar{T}(x, s)(\mathbf{x} \cdot \mathbf{v}) \partial_{s} \zeta(x, s) d x d s\right\rangle \\
& =\kappa_{0}\left\langle\int_{0}^{t} \int_{D}(\mathbf{x} \cdot \mathbf{v}) \nabla \zeta(x, s) \cdot \overline{\mathbf{q}}(x, s) d x d s\right\rangle \\
& \quad+\kappa_{0}\left\langle\int_{0}^{t} \int_{D} \zeta(x, s) \mathbf{v}^{T} \cdot \overline{\mathbf{q}}(x, s) d x d s\right\rangle .
\end{aligned}
$$

Thus, we reach the conclusion that

$$
\begin{aligned}
\kappa_{0} & \left\langle\int_{0}^{t} \int_{D} \zeta(x, s) \mathbf{v}^{T} \cdot \overline{\mathbf{q}}(x, s) d x d s\right\rangle \\
& =-\left\langle\int_{0}^{t} \int_{D}(\nabla \zeta(x, s))^{T} \cdot{\underset{\sim}{*}}^{*} \cdot \mathbf{v} \bar{T}(x, s) d x d s\right\rangle .
\end{aligned}
$$

Integration by parts in (A.72) leads to the desired result:

$$
\kappa_{0} \overline{\mathbf{q}}(x, s)=\underset{\sim}{\kappa} * \cdot \nabla \bar{T}(x, s)
$$


Finally, note that (A.73) holds true along an arbitrary weakly convergent subsequence $T_{\delta_{n}}(x, s)$, so that, in fact, we have $\lim _{\delta \downarrow 0} T_{\delta}(x, s)=\bar{T}(x, s)$, for $0<\delta<1$. This concludes the proof of the homogenization theorem. We note here that this theorem provides an a posteriori justification of the fact that the effective diffusivity tensor $\underset{\sim}{\kappa} *$ corresponding to (1) is uniquely determined by the formula

$$
\stackrel{\kappa}{\kappa}^{*}=\langle(\mathbf{I}+P e \mathbf{H}) \cdot \mathbf{E}\rangle=\lim _{j>\infty}\left\langle\left(\mathbf{I}+P e \mathbf{H}^{\left(n_{j}\right)}\right) \mathbf{E}^{\left(n_{j}\right)}\right),
$$

independently of which particular subsequence $\left\{\mathbf{E}^{\left(n_{J}\right)}\right\}$, converging weakly in $L^{2}(\langle\cdot\rangle)$ is chosen in $(2.31)$.

\section{References}

1. Csanady, G.T.: Turbulent diffusion in the environment. Geophysics and Astrophysics Monographs, Dordrecht, Holland: D. Reidel 1973

2. Young, W., Pumir, A., Pomeau, Y.: Phys. Fluids A1, 462 (1989)

3. Koch, D.L., Cox, R.G., Brenner, H., Brady, J.F.: J. Fluid Mech. 200, 173-178 (1989)

4. Krommes, J.A., Smith, R.A.: Ann. Phys. 177, 246 (1987)

5. Mathéron, G., De Marsily, G.: Water resources research, Vol. 16 (5), 901-917 (1980)

6. Bouchaud, J.P., Comptet, A., Georges, A., Le Doussal, P.: J. Phys. (Paris) 48, 1445 (1987)

7. Avellaneda, M., Majda, A.J.: Mathematical models with exact renormalization for turbulent transport. Commun. Math. Phys. 131, 381-429 (1990)

8. Kubo, R.: Math. Phys. 4, 174 (1963)

9. McLaughlin, D., Papanicolaou, G.C., Pironneau, O.: SIAM J. Appl. Math. 45, 780 (1985)

10. Papanicolaou, G.C., Varadhan, S.R.S.: In: Random fields, Coll. Math. Soc. Janos Bolyai, Vol. 27, pp. 835-873. Fritz, J., Lebowitz, J.L., Szasz, D. (eds.). Amsterdam: North-Holland 1982

11. Tartar, L.: Cours Peccot. Collège de France, 1977-1978

12. Bensoussan, A., Lions, J.L., Papanicolaou, G.C.: Asymptotic analysis for periodic structures. Amsterdam: North-Holland 1978

13. Oelschlager, K.: Ann. Prob. 16 (3), 1084-1126 (1988)

14. Osada, H.: In: Proceedings Fourth Japanese-USSR Symp. in Prob. Theory. Lecture Notes in Mathematics, Vol. 1021, pp. 507-517. Berlin Heidelberg New York: Springer 1983

15. Kraichnan, R.: Complex Systems 1, 805-820 (1987)

16. Yakhot, V., Orszag, S.: Analysis of the $\varepsilon$-expansion in turbulence theory: approximate renormalization group for diffusion of a passive scalar in a random velocity field. Preprint, October 1988

17. Baker, Jr., G.A., Gammel, J.L.: The Padé approximant in theoretical physics. New York: Academic Press 1970

18. Childress, S., Soward, A.: Scalar transport and alpha-effect for a family of cat's eye flows. Preprint, Sept. 1988

19. Golden, K., Papanicolaou, G.C.: Commun. Math. Phys. 90, 473 (1983)

20. Berman, D.: Phys. Rep. Phys. Lett. C43, 377 (1978)

21. Milton, G.W.: J. Appl. Phys. 52, 5286 (1982)

22. Battacharya, R., Gupta, V., Walker, H.F.: SIAM J. Appl. Math. 49, 1 (1989)

23. Wolynes, P.G.: Phys. Rev. A11, 1700 (1975)

24. Kraichnan, R.: In: ref. 17, p. 129

25. Hashin, Z., Shtrikman, S.J.: Mech. Phys. Solids 11, 127-140 (1963)

26. Schulgasser, K.: J. Appl. Phys. 54, 1, 380 (1982)

27. Avellaneda, M., Majda, A.J.: Phys. Rev. Lett. 62, 753 (1989)

28. Beran, M.: Statistical continuum theories. New York: Wiley 1968

29. Riesz, F., Nagy, B. Sz.: Leçons d'Analyse Fonctionelle. Paris-Budapest: Gauthier-VillarsAkademi Kiado 1952 
30. Widder, D.V.: The Laplace transform. Princeton, NJ: Princeton University Press 1946

31. Bayly, B.: Ph. D. Thesis, Princeton University, 1986

32. Wheeler, J.C., Gordon, R.G.: In ref. 17, p. 99

33. Schulgasser, K.: J. Phys. C10, 407 (1977)

34. Avellaneda, M., Cherkaev, A.V., Lurie, K.A., Milton, G.W.: J. Appl. Phys. 63 (10), 49895003 (1988)

35. Avellaneda, M., Milton, G.W.: SIAM J. Appl. Math. 49 (3), 824-837 (1989)

36. Avellaneda, M.: Commun. Pure Appl. Math. 4, 527 (1987)

37. Kim, C.B., Krommes, J.A.: J. Stat. Phys. 59, 5/6, 1103 (1988)

38. Deutscher, G., Zallen, R., Adler, D.: Percolation structures and processes. Am. Israel Phys. Soc. 5, 297-321 (1983)

39. Kesten, H.: Percolation theory for mathematicians. Boston: Birkhäuser 1982

40. Nash, J.: Am. J. Math. 80, 931-954 (1958)

41. Strook, D., Varadhan, S.R.S.: Multidimensional diffusion processes. Berlin Heidelberg New York: Springer 1979

42. Ladyženskaja, O.A., Solonnikov, V.A., Ural'ceva, N.N.: Linear and quasilinear equations of parabolic type. Providence, RI: Am. Math. Soc. 1968

43. Shraiman, B.: Phys. Rev. A36, 261 (1987)

44. Rosenbluth, M.N., Berk, H.L., Doxas, I., Horton, W.: Phys. Fluids 30 (9), 2636 (1987)

45. Avellaneda, M., Kim, I.C., Torquato, S.: To appear in Phys. Fluids A, 1991

Communicated by S.-T. Yau 
